\title{
AUTHOR INDEX (Volume 8)
}

Abada, A., Bougourzi, A.H. \& El Gradechi, M.A., Deformation of the Wakimoto construction Abe, K., see Elia

Abt, I., see Elia

Abu Leil, G.F., Soft photon radiation in $e^{+} e^{-} \rightarrow t \bar{t} \rightarrow$ $b \bar{b} W^{k} W^{\prime \prime}$

Achasov, N.N., \& Shestakov, G.N., Towards the determination of the $\pi p \rightarrow f_{2}(1270) n$ reaction crosis-section

Acton, P.D., se' Elia

Adamovich, M.I., 't al., EMU0] Collaboration, Study of angulat distribution of helium projectile fragments in interactions of $200 \mathrm{~A} \mathrm{GeV}{ }^{32} \mathrm{~S}$ ions with emulsion nuclex

Adel, K., \& Yao, Y.-P., Effective Lagrangian for $b \rightarrow s$ processes with QCD-corrections

Adolphisen, C.F., se'e Elia

Aggarwal, M.M., s'c' Adanovich

Aghamohammadi, A., The twoparametric exlension of $h$ deformation of (iL.(2), and the differential calculus on its quantum plane

Agnew, G., s'c' Elia

Aharonov, Y., Popescu, S. \& Rohrlich, D., Algebraic approach to the Born-Oppenheimer approximation

Ahluwalia, D.V., \& Goldman, T', Space-time symmetries and vorlices in the cosmos

Almad, T., Nasr, M.A. \& Irfan, M., Multiparticle production in silicon-emulsion interactions at $4.5 \mathrm{~A} \mathrm{GeV} / \mathrm{c}$

Akdeniz, K.G., Dayi, OJ.F, \& Kizilersü, A.. Reply to the comment

Alber, $C ., x^{\prime} e^{\prime}$ Ellia

Aleixo, A.N.F., Bertulani, C.A. \& Hussein, M.S., Heavy ion excitation of giant resonances: a bridge from the elastic scattering to the inelastic data

Alexandrov, Y.A., see Adamovich

Ali, A., \& Kumar, A., A new $N=4$ superconl'ormal algebra

Ali, A., \& Kumar, A., $O(d, \bar{d})$ trunsformations and $3 \mathrm{D}$ black hole

Alkoter, R., see Weiss
Allen, TJ., Collective coordinate action for charged sigmamodel vortices in finite geometries

A8 (1993) 715

A8 (1993) 2237

A8 (1993) 2237

Alzetta, R., Liberti, G., Gibilisco, M. \& Preparata, G., A weak decays in medium to heavy hypernuclei

A8 (1993) 1191

Alzoton, D.F., see Elia

Ambjorn, J., \& Kristjansen, C.F. From one-matrix model to Kontsevich model

A8 (1993) 2343

A8 (1993) 2237

Anada, H., Kitazoe, T. \& Mizunoto, Y., Perturbutive approach to the WheelerDeWitt equation

A8 (1993) 1815

A.8 (1993) 2335

A8 (1993) 2237

A8 (1993) 2875

A8 (1993) 45. $1065(\mathrm{E})$

Anderson, J.T., Transtormation to autonomous systems via Hamilton's equations

A8 (1993) 233

A8 (1993) 21

A8 (1993) 1679

A8 (1993) 2237

A8 (1993) 21

Andreeva, N.P., see Adamovich Andrianov, V.A., \& Manashov, A.N., Asymptotic restrictions and the generalized Skyrme models

Antillon, A., see Escalona

Antilogus, P., see Elia

Antoniadis, I., \& Odintsov, S.D.,

A8 (1993) 2607

A8 (1993) 2237

Conformal dynamics of quantum gravity with torsion

Antunovic, $\breve{Z}$., Blagojević, M. \& Vukašinac, T., BRST structure and auxiliary fields of simple supergravity

Anzon, Z.V., see Adamovich

Aoki, S., \& Kikukawa, Y.. Anomaly through gatuge-invariant regularization with infinite number of Pauli-Villars fields

A8 (1993) 1103 Aouissat, Z., Chanfray, G. \& Schuck, P., Influence of the particle-hole continuum on pion-pion correlations in dense nuclear matter

A8 (1993) 2905

A8 (1993) 22,37

Aoyama, S., \& Vandoren, S. The Batalin-Vilkovisky formalism on fermionic Kaihler manifolds

A8 (1993) 997

Aratyn, H., Ferreira, L.A., Gomes, J.F., Medeiros, R.T. \& Zimerman, A.H., Generalized Miura transformations, twoboson KP hierarchies and their reduction to $\mathrm{KdV}$ hierarchies

Aref"eva, I.Ya., \& Zubarev, A.P., Divergences of discrete states amplitudes and effective Lagrangian in 2D string theory A8 (1993) 1469 
Aref"eva, 1.Ya. Medvedev, P.B. \& Zubarev, A.P., Interaction of $d=2, c=1$ discrete states from string field theory

Arnaudov, L.N., Prodanov, E.M. \& Rashkov, R.Ch., 2D supergravity with applications to integrable models

Arora, R., see Adamovich

Arriola, E.R., \& Salcedo, L.L. Semiclassical expansion for Dirac Hamiltonians

Artuyo, C., sec Elia

Ash, W.W., see Eliat

Asthford, V., se'e Elia

Askew, A.J., Kwiecinski, J. Martin, A.D. \& Sutton, P.J. A structure function test of the QCD Lipatov behavior at HERA

Astbury, A., see Elia

Aston. D, see Elia

Au, Y., se' Elia

Avetyan, F.A. sere Adamovich

Awada, M., Ma, M. \& Zoller, D.

Rigid QED and nonlinear sigma models

Axen, D.A., se Elia

Azcoiti, V., \& Luo, X.-Q., Phase structure of compact lattice $\mathrm{OED}_{3}$ with massless fermions

Baacke, J., \& Junker, S., Quantum corrections to the electroweak sphateron transition

Baaquie, B.E., \& Rajeev, S.G. Long distance behavior of the four-dimensional nonlinear signa model with anomaly

Baaquie, B.E., Feynman path-integral for the sum of the globa and local Kac-Moody characters

Bacchetta, N., sec Elia

Badyal, S.K., se' Adamovich

Baird, K.G., st't' Elia

Baker, W., se Elia

Ballachandran, A.P., Bimonte, G. \& Teotonio-Sobrinho, P., Edge states in $4 D$ and their $3 D$ groups and fields

Baltay, C., se'e Elial

Band. H.R. se' Elia

Bando, M., \& Matekawa, N., A signal for technicolor?

Bando, M. Matekawa, N. Nakano, H. \& Sato, J., A handy calculating method of Higgs potential in SUSY model

Banerjee, P,K., se' Maity
A8 (1093) 2167

A8 (1993) 2943

A8 (1993) 21

A8 (1993) 2061

A8 (1993) 2237

A8 (1993) 2237

A8 $(1993) 2237$

A8 (1993) 3813

A8 (1903) 2237

A8 (1993) 2237

A8 ( 5993$) 2237$

A8 (1993) 21

A8 (1993) 2585

A8 (1993) 2237

A8 (1993) 36.35

A8 (1993) 2869

A8 (1993)625

A8 (1993) 2449

A8 (1993) 2237

A8 (1993) 21

A8 (1993) 2237

A8 (1943) 2237

A8 (1993) 1305 A8 (1993) 2237 A8 (1993) 2237

A8 (1993) 2141

A8 (1993) 2729 A8 (1903) 38.53
Baranko, G. see Elia

Bardon, O., see Elia

Barrera, F., see Elia

Barut, A.O., \& Zino, G., On parity conservation and the question of the 'missing' (righthanded) neutrino

Barut, A.O., Shnir, Ya.M. \& Tolkachev, E.A., The Lamb shift in the charge-magnetic monopole system

Barut, A.O., \& Roy, P., Dynamical groups and supersymmetry II. Supersymmetric Aharonov. Bohm and anyon systems

Basova, E. sec Adamovich

Bastrukov, S.I., On elastic vibra tions of homogeneous star

Batakis, N.A., \& Kehagias, A.A., Topological obstructions to BRST invariance

Batalin, I.A. \& Tyutin, I.V., On the multilevel generalization of the field-antifield formalism

Batalin, I.A., \& Tyutin, I.V., On the equivalence between the unified and standard versions of constraint dynamics

Battiston, R., see Elia

Bazarko, A.O., see Elia

Bean, A., see Elia

Becker, K., \& Becker, M., Nonperturbative solation of the super-Virasoro constraints

Becker, M., se' Becker

Beckers, J., Debergh, N, \& Nikitin, A.G., More on parasupersymmetries of the Schrödinger equation

Bedaque, P.F., \& Dats, A., Oul of equilibrium phase transitions and a toy model for disoriented chiral condensittes

Beer, G., see Elia

Belcinski, R.J. $5 e^{\circ}$ Elia

Belgiorno, F., Cattaneo, A.S. Fucito, F. \& Martellini, M., A conformal affine Toda model of $2 \mathrm{D}$ black holes: $\mathrm{A}$ quantum study of the evaporation end point

Belokurov, V.V \& Iofa, MZ Ultraviolet finite gauge theories in the live-dinensional space-time

Belov, A.A., \& Claltikian, K.D. Q-deformation of Virasoro algebra and lattice conformal theories

Ben-David, R., sec Elial
A8 (1993) 2593

A8 (1993) 2237

A8 (1993) 2237

A8 (1993) 2237

A8 (1993) 2237

A8 (1993) 1011

A8 (1993) 3443

A8 (1993) 3507

A8 (1993) 21

A8 (1993) 711

A8 (1993) 30.57

A8 (1993) 3673

A8 (1993) 3757

A8 (1993) 2237

A8 (1993) 2237

A8 (1993) 2237

A8 (1993) 1205

A8 (1993) 1205

A8 (1993) 435

A8 (1993) 3151

A8 (1993) 2237

As (1993) 2237

A8 (1903) 3345

A8 (1903) 2237
A8 (1993) 1233 
Benvenuti, A.C., see Elia

Berger, R., see Elia

Berridge, S.C., see Elia

Bertulani, C.A., see Aleixo

Bethke, S., see Elia

Beyer, R., see Rädel

Bhalla, K.B., see Adamovich

Bhasin, A., see Adamovich

Bhasin, V.S., see Parashar

Bhatia, V.S., see Adamovich

Bhattacharjee, D.K., see Maity

Bhattacharya, B., see Chatterjee

Bhawal, B., \& Mani. H.S., Semiclassical decay of Kaluza-Klein vacuum in higher derivative gravity

Bian, J.-G, \& Huang, Tao, Pseudoscalar decay constants and mass spectrum of $q \bar{q}$ and $Q \ddot{q}$ system in QCD theory involving the vacuum condensates

Biasini, M., sec Elia

Bienz, T., see Elia

Bilei, G.M., seo Elia

Bimonte, G., s'e' Balachandran

Bird, F., see Elia

Bisello, D., see Elia

Blagojevic, M., Sazdovie, B. \& Vukašinac, T., SUSY auxiliary fields from BRST analysis

Blagojevic, M,, se' Antunovic

Blaylock, G., sec Elia

Blumberg, R., see Elia

Bogart, J.R., see' Elia

Bogdanov, V.G., sue Adamovich

Bolton, T. see Elia

Boos, H.E., Equivalence of $S_{3}$ invariant lattice model and model of vertex-lace type

Bougerolle, S., sece Elia

Bougourzi. A.H., see Abada

Boulatov, D.V., Infinite-tension strings at $d>1$

Boulatev, D.V., $q \mathrm{QCD}_{2}$ and $G / G$ model

Bower, G.R., see Elia

Boyce, R.F., sice Elia

Brahmachari, B., Patra, P.K., Sarkar, U. \& Sridhar, K., Higher-dimensional operators to the rescue of minimal $S U(5)$

Brahmachari, B, Sarkar, U, \& Sridhar, K., Non-perturbative unilication in the light of LEP resuits

Bramon, A., see Karlsen

Bratchikov, A.V., Imbedding of transformation groups in affine ones and renormalization of two-dimensional homogeneous $\sigma$-models
A8 (1993) 2237

A8 (1993) 2237

A8 (1993) 2237

A8 (1993) 997

A8 (1993) 2237

A8 (1993) 1067

A8 (1993) 21

A8 (199.3) 21

A8 (1993) 389 ,

2525(E)

A8 (1993) 21

A8 (1993) 3853

A8 (1993) 2249

A8 (1993) 1621

A8 (1993) 635

A8 (1993) 2237

A8 (1993) 2237

A8 (1993) 2237

A8 (1993) 1305

A8 (1993) 2237

A8 (1993) 2237

A8 (1993) 349

A8 (1993) 1983

A8 (1993) 2237

A8 (1993) 2237

A8 (1993) 2237

A8 (1993) 21

A8 (1993) 2237

A8 (1993) 3139

A8 (1993) 2237

A8 (1993) 715

A8 (1993) 557

A8 (1993) 3491

A8 (1993) 2237

A8 (1993) 2237

A8 (1993) 1487

A8 (1993) 3349 A8 (1993) 97

A8 (1993) 2937
Brau, J.E. see Elia

Breidenbach, M, see Elia

Brink, L., \& Vasiliev, M.A.,

Representations of the $S_{N}$ extended Heisenberg algebra and relations between Knizhnik-Zamolodchikov equations and quantum Calogero model

Brooks, R., Diff( $\Sigma)$ and metrics from Hamiltonian-TQFT's in $2+1$ dimensions

Browder, T.E., see Elia

Brunelli, J.C., \& Mendes, R.S. Stochastic quantization and $1 / N$ expansion

Brunini, S.A., \& Gomes, M., The Gauss-Bonnet identity in fourth order gravity

Brzeziński, T., Rembieliński, J.

\& Smoliński, K.A., Quantum particle on a quantum circle

Bubnov, V.I., see Adamovich

Buccella, F., \& Soffer, J., Parton distributions in the nucleon and the Pauli principle

Bugg, W.M., see Elia

Burgess, B., see Elia

Burke, D., see Elia

Burnett, T.H., see Adamovich

Burnett, T.H., see Elia

Burrows, P.N., see Elia

Busza, W., see Elia

Byers, B.L., see Elia

Bytsenko, A., Kirsten, K. \& Odintsov, S., Self-interacting scalar fields on space-time with compact hyperbolic spatial part

Bytsenko, A.A., Elizalde, E. Odintsov, S.D. \& Zerbini, S., A finite temperature periodic structure in (super)string theory

A8 (1993) 2237

A8 (1993) 2237

A8 (1993) 3585

A8 (1993) 2277

A8 (1993) 2237

A8 (1993) 115

A8 (1993) 1977

A8 (1993) 409

A8 (1993) 21

A8 (1993) 225

A8 (1993) 2237

A8 (1993) 2237

A8 (1993) 2237

A8 (1993) 21

A8 (1993) 2237

A8 (1993) 2237

A8 (1993) 2237

A8 (1993) 2237

A8 (1993) 2011

A8 (1993) 1131

Bytsenko, A.A., \& Zerbini, S. Semiclassical approximation for a class of quantum $p$-brane models

Caenepeel, D., \& Mackenzie, R., Parity violation and the mean field approximation for the anyon gas

Cai, X., see Adamovich

Calcaterra, A., see Elia

Caldwell, D.O., see Elia

Calloway, D., see Elia

Camanzi, B., see Elia

Camilleri, 1., see Elia

Carpinelli, M., sec Elia

Carr, J., see Elia

Cartwright, S., se' Elia

Cassell, R., see Elia

Castaldi, R., se' Elia
A8 (1993) 1573

A8 (1993) 1909

A8 (1993) 21

A8 (1993) 2237

A8 (1993) 2237

A8 (1993) 2237

A8 (1993) 2237

A8 (1993) 2237

A8 (1993) 22.37

A8 (1993) 2237

A8 (1993) 2237

Ax (1993) 2237

A8 (1993) 2237 
Castro, A., see Elia

Cattaneo, A.S., see Belgiomo

Cavalli-Sforza, M., see Elia

Cavicchi, M., di Vecchia, P. \&

Pesando, I., The master field of $\mathrm{QCD}_{2}$ and the 't Hooft equation

Chadwick, G.B., see Elia

Chakrabarti, R., Jagannathan, R

\& Vasudevan, R., A new look at the $q$-deformed calculus

Chakraborty, A., see Chakraborty

Chakraborty, S., \& Chakraborty, A., Inhomogeneous string cosmology in higher dimension

Chaltikian, K.D., see Belov

Chamberlain, O., see Elia

Chambers, D., see Elia

Chanfray, G., see Aouissat

Chao, Liu, Light-cone quantization of scalar field

Chasnikov, I.Y., see Adamovich

Chatterjee, P., \& Bhattacharya

B., Space-time torsion, broken Lorentz symmetry and inflation in the early universe

Chaturvedi, S., see Shanta

Chaturvedi, S., Jagannathan, R. \& Srinivasan, V., TammDancoff deformation of bosonic oscillator algebras

Chaudhuri, S., \& Minic, D., A note on effective string theory Chen, L., see Elia

Chernova, L.P., see Adamovich

Chernyausky, M.M., sec Adamovich

Chetyrkin, K.G., Kwiatkowski, A. \& Steinhauser, M., Leading top mass corrections of order $O\left(\alpha \alpha_{s} m_{i}^{2} / M_{W}^{2}\right)$ to the partial decay rate $\Gamma(Z \rightarrow b \bar{b})$

Chiang, H.C., \& Oset, E., Back ground for $\left(\mu^{-}, e^{-}\right)$conver sion in nuclei from muon decay in orbit

Chikalov, V., \& Pashnev, A. Twistor-like type Il superstring and bosonic string

Chiu, T.W., \& Lee, H.C., Quantum holonomy on a lattice

Chizhov, M.V., New tensor particles from $\pi^{-} \rightarrow e^{-} \tilde{v} \gamma$ and $K^{+} \rightarrow \pi^{\circ} e^{+} v$ decays

Choi, J.M., see Myung

Chou, $C_{.}$, On the quantization of damped systems

Chung, B.K., Joo, K.G. \& Nam, S., Hamiltonian formulation of SL(3) Ur-KdV equation
A8 (1993) 2237

A8 (1993) 2593

A8 (1993) 2237

A8 (1993) 2427. 2909(E)

A8 (1993) 2237

A8 (1993) 2695

A8 (1993) 889

A8 (1993) 889

A8 (1993) 1233

A8 (1993) 2237

A8 (1993) 2237

A8 (1993) 1379

A8 (1993) 3165 A8 (1993) 21

A8 (1993) 2249 A8 (1993) 1999

A8 (1993) 3727

A8 (1993) 2763 A8 (1993) 2237

A8 (1993) 21

A8 (1993) 21

A8 (1993) 2785

A8 (1993) 2259

A8 (1993) 285

A8 (1993) 2911

A8 (1993) 2753 A8 (1993) 341

A8 (1993) 2037

A8 (1993) 2927
Chung, W.-S., q-deformed Baker-Campbell-Hausdorff formula

Clarke, P.E.L., see Elia

Claus, R., see Elia

Clendenin, J., see Elia

Cline, D.B., see Hong

Cline, D.B. Rare FCNC-B decays at hadron machines

Cohn, H.O., see Elia

Cohn, J.D., \& Periwal, V., The strength of string nonperturbative eflects and strong-weak coupling duality

Coller, J.A., see Elia

Cook, V., se'e Elia

Cords, D., see Elia

Cotton, R., see Elia

Cowan, R.F., see Elia

Coyle, P.A., see Elia

Coyne, D.G., see Elia

Craddock, W., see Elia

Crescimanno, M., Handle operators of coset models

Cutler, $\mathrm{H}$, see Elia

D'Oliveira, A., see Elia

Damerell, C.J.S., see Elia

Danielsson, U.H., Black hole uncertainties

Das, A., \& Galvão, C.A.P., Selfduality and the KdV hierarchy

Das, A., \& Galvão, C.A.P., Selfduality and the supersymmetric KdV hierarchy

Das, A., \& Mathur, V.S., Nonleptonic decays of charmed mesons into two pseudoscalar mesons

Das, A., see Bedaque

Das, B.B., sec Maity

Das, S.R., Matrix models and black holes

Das, S.R., Matrix models and non-perturbative string propagation in two-dimensional black hole backgrounds

Dasu, S., see Elia

Datta, D.P., Semiclassical backreaction and Berry's phase

A8 (1993) 2569

A8 (1993) 2237

A8 (1993) 2237

A8 (1993) 2237

A8 (1993) 146

A8 (1993) 3397

A8 (1903) 2237

A8 (1993) 3659

A8 (1993) 2237

A8 (1993) 2237

A8 (1993) 2237

A8 (1993) 2237

A8 (1993) 2237

A8 (1993) 2237

A8 (1993) 2237

A8 (1993) 2237

A8 (1993) 1877

A8 (1993) 2237

A8 (1993) 2237

A8 (1993) 2237

A8 (1993) 1925

A8 (1993) 661

A8 (1993) 1390

A8 (1993) 2079

A8 (1993) 3151

A8 (1993) 3853

A8 (1993) 69

A8 (1993) 1331

A8 (1993) 2237

As(1093) 191 2523(E)

Datta, D.P., Berry's phass and energy conservation in a grayity-scalar system

Davis, R., sec Elia

Dayi, Ö.F., A general solution of the master equation for a class of first order systems

Dayi, O.F., A general solution of the BV-master equation and BRST field theories
A8 (199.3) 60)

A8 (1993) 811

A8 (1093) 2087
A8 (1993) 2237 
Dayi, Ö.F., see Akdeniz

de Sangro, R., see Elia de Simone, P., see Elia de Simone, S., see Elia

De, T., see Kundu

Dean, T., see Elia

Debergh, N., see Beckers

Debergh, N., A note on genon and parastatistics

Decker, R.J., see Elia

Degl'Inrocenti, S., \& Ricei, B. Bounds on lepton flavo changing currents and the solar neutrino puzzle

DeJongh, F., see Elia

Delbecq, C., \& Quesne, C., A cubic deformation of $\mathrm{su}(2)$

Dell'orso, R, st'e' Elia

Demers, J.-G., Dynamical supersymmetry and solutions for Pauli Hamiltonians

Destri, C., see Maraner

Dhar, A., Mandal, G. \& Wadia,

S.R., Wave propagation in stringy black hole

Dhar, A., Mandal, G. \& Wadia,

S.R., $W_{x}$ eoherent states and path-integral derivation of bosonization of non-relativistic fermions in one dimension

di Vecchia, P., see Cavicchi

Dickey, L.A., Additional symmetries of KP. Grassmannian, and the string equation

Dickey, L.A., Additional symmetries of KP, Grassmannian, and the string equation II

Disco, A., see Elia

Dobroliubov, M.I., \& Ignatiev, A.Yu., Do millicharged particles exist?

Dobroliubov, MI, Eliezer, D Kogan, I.I., Semenoff, G.W. \& Szabo, R.J., The spectrum of topologically massive quantum electrodynamics

Dobroliubov, M.I., Makeenko, Yu. \& Semenoff, G.W Correlators of the Kazakov Migdal model

Dolan, B.P., Potential flow of the renormalization group in a simple two-component model

Dolin, R., see Eli

Downing, R.W., see Elia

Dowrick, N.J., \& McDougall, N.A., The analytic propertic of the quark propagator in the instanton dilute-gas approximation
A8 (1993) 2905
A8 (1993) 2237
A8 (1993) 2237
A8 (1993) 2237
A8 (1993) 2465
A8 (1993) 2237
A8 (1993) 435

A8 (1993) 765

A8 (1993) 2237

A8 (1993) 471

A8 (1993) 2237

A8 (1993) 961

A8 (1993) 2237

A8 (1993) 827

A8 (1993) 861

A8 (1993) 1701

A8 (1993) 3557

A8 (1993) 2427

2909(E)

A8 (1993) 1259

A8 (1993) 1357 A8 (1993) 2237

A8 (1993) 917

A8 (1993) 2177

A8 (1993) 2387

A8 (1993) 3103 A8 (1993) 2237 A8 (1993) 2237

A8 (1993) 503
Dreiner, H., Leontaris, G.K. \& Tracas, N.D., Ansatz for quark, charged lepton, and neutrino masses in SUSY GUTs

Dremin, I.M., New analysis of multiplicity distributions

A8 (1993) 2099

Du, D., \& Liu, C., Universal Yukawa interactions in supersymmetry

Du, Y.C., see Elia

Dubois, R., see Elia

Duboscq, J.E., see Elia

Dumitru, A., Rischke, D.H., Stöcker, H. \& Greiner, W. Photon suppression in a baryon-rich plasma

Dunbar, D.C., \& Joshi, K.G., Maverick examples of coset conformal field theories

Dunwoodie, W. see Elia

Durand, S., Extended fractional supersymmetric quantum mechanics

Durand, S., Fractional superspace formulation of generalized mechanics

Durrett, D.D., see Elia

Dutta-Roy, B., \& Ghosh, G., Curvature of Hilbert space and $q$-deformed 'quantum mechanics'

Dutta-Roy, B., see Kundu

Duval, C., Horváth, $Z$. \& Horvathy, P.A., Strings in plane-fronted gravitational waves

Dvoeglazov, V.V., Faustov, R.N. \& Tyukhtyaev, Y.N., Decay rate of a positronium. Review of theory and experiment

Ecklund, S.D., see Elia

Edelstein, J.D., Lozano, G. \& Schaposnik, F.A., Vortices in curved space-time

Eguchi, T., Yamada, Y, \& Yang, S.-K., Topological field theories and the period integrats

Eholzer, W., Honecker, A. \& Hibbel, R., Representations of $N=1$ extended superconformal algebras with two generators

Eigen, G., see Elia

Eisenstein, B.I., see Elia

El Gradechi, M.A., see Abada

Elia, R., et al., SLD Collaboration, First measurement of the left-right asymmetry in $Z$. boson production

A8 (1993) 2747

A8 (1993) 2271

A8 (1993) 2237

A8 (1993) 2237

A8 (1993) 2237

A8 (1993) 1291

A8 (1993) 2803

A8 (1993) 2237

A8 (1993) 1795

A8 (1993) 2323

A8 (1993) 2237

A8 (1993) 1427

A8 (1993) 2465

A8 (1993) 3749

A8 (1993) 3263

A8 (1993) 2237

A8 (1993) 3665

A8 (1993) 1627

A8 (1993) 725

A8 (1993) 2237

A8 (1993) 2237

A8 (1993) 715

A8 (1993) 2237 
Eliashvili, M,, \& Tsitsishvili, G,, On the current correlators for planar fermions in magnetic field

Eliezer, D., see Dobroliubov

Eligbaeva, G.Z., see Adamovich

Elizalde, E., \& Odintsov, S.D., One-loop divergences in twodimensional Maxwell-Dilaton quantum gravity

Elizalde, E., see Bytsenko

Elizalde, E., \& Odintsov, S.D., Gravitational phase transitions in infrared quanturn gravity

Ellis, J., Unification and pluralism in theoretical physics

Emma, P.J., see Elia

Emmet, II, W.T., see Elia

Emparan, R., \& Valle Basagoiti, M.A., Three-loop calculation of the anyonic full cluster expansion

English, R.L., see Elia

Erdos, E., see Elia

Eremenko, L.E., see Adamovich

Escalera, J., see Elia

Escalona, J., Torres, M. \& Antillon, A., Stability of nontopological Chern-Simons vortices in a $\phi^{2}$-model

Esposito, S., The solar neutrino problem within MSW theory

Faber, M., Ivanov, A.N., Nagy, M. \& Troitskaya, N.I., Chiral symmetry breaking and the gluon condensate

Faber, M., see Ivanov

Fuessler, A., see Nojarov

Fakir, R., \& Habib, S., Quantum fluctuations with strong curvature coupling

Falbo-Kenkel, M.K., see Mansouri

Fan, C., see Elia

Fang, L.-Z., Size of multiplyconnected universe and cosmic background radiation

Faustov, R.N., see Dvoeglazov

Fayyazuddin, A., Exact ground state wave functions for $N$ anyons in a magnetic field on a torus

Feng, D.H., see Zhang

Fero, M.J., see Elia

Ferrara, S., \& Porrati, M., String phase transitions in a strong magnetic field

Ferreira, L.A., see Aratyn

Ferrie, J., see Elia

Fieguth, T., see Elia
A8 (1993) 3807

A8 (1993) 2177

A8 (1993) 21

A8 (1993) 33

A8 (1993) 1131

A8 (1993) 3325

A8 (1993) 1

A8 (1993) 2237

A8 (1993) 2237

A8 (1993) 3291

A8 (1993) 2237

A8 (1993) 2237

A8 (1993) 21

A8 (1993) 2237

A8 (1993) 2955

A8 (1993) 1557

A8 (1993) 335

A8 (1993) 1021

A8 (1993) 1171

A8 (1993) 2827

A8 (1993) 2503

A8 (1993) 22.37

A8 (1993) 2615 A8 (1993) 3263

A8 (1993) 3173

A8 (1993) 1417

A8 (1993) 2237

A8 (1993) 2497

A8 (1993) 3079

A8 (1993) 2237

A8 (1993) 2237
Figueroa-O'Farrill, J.M., \& Stanciu, S., New supersymmetrizations of the generalized KdV hierarchies

Filho, E.D., Supersymmetric solution for two-dimensional Schrödinger equation

Finch, A., Jet production in $\gamma \gamma$ physics

Flynn, J., see Elia

Foot, R., Lew, H. \& Volkas, R.R., Are quarks and leptons related by $C, P$ and $T$ symmetries?

Foot, R., \& Lew, H., Minicharged tau neutrinos?

Forbush, D.A., see Elia

Forger, M., \& Laartz, J., The algebra of the energy-momentum tensor and the Noether currents in off-critical NZNW models

Fortune, K.M., see Elia

Fox, J.D., see Elia

Fox, M.J., see Elia

Frederico, T., \& Hussein, M.S., The structure of ${ }^{11} \mathrm{Li}:$ a "Rydberg" nucleus?

Freire, F., see O'Connor

Frey, R, see Elia

Freytag, D.R., see Elia

Friedman, J.I., see Elia

Frisch, J., see Elia

Fucito, F., see Belgiorno

Fujii, A., see Quano

Fujimoto, J., see Elia

Fujishiro, T.T., Hayashi, M.J. \& Takeshita, S., The cosmic strings generated from the torsion

Fujitsu, A., Free field representation of the $N=3$ superconformal algebra from Hamil. tonian reduction of $\operatorname{osp}(3 / 2)$ affine Lie superalgebra

Fujiwara, T., \& Kitakado, S. Topless electroweak model as anomalous gauge theory

Fujiwara, T., Tabei, T., Igarashi, Y., Máda, K. \& Kubo, J. Canonical treatment of two-dimensional gravity as an anomalous gauge theory

Furuno, K., see Elia

Gaillard, M., see Elia

Gaitinov, A.S., see Adamovich

Gallinaro, M., see Elia

Galvão, C.A.P., see Das

Galvāo, C.A.P., see Das
A8 (1993) 2125

A8 (1993) 63

A8 (1993) 3303

A8 (1993) 2237

A8 (1993) 1859

A8 (1993) 3767

A8 (1993) 2237

A8 (1993) 803

A8 (1993) 2237

A8 (1993) 2237

A8 (1993) 2237

A8 (1993) 295

A8 (1993) 1779

A8 (1993) 2237

A8 (1993) 2237

A8 (1993) 2237

A8 (1993) 2237

A8 (1993) 2593

A8 (1993) 1585

A8 (1993) 2237

A8 (1993) 491

A8 (1993) 1763

A8 (1993) 1639

A8 (1993) 2147

A8 (1993) 2237

A8 (1993) 2237

A8 (1993) 21

A8 (1993) 2237

A8 (1993) 661

A8 (1993) 1390 
Gambhir, Y.K., \& Ring, P., Recipe for the solution of the Dirac equation by the basis set expansion method

Ganssauge, E.R., see Adamovich

Garpman, S., see Adamovich

Garwin, E., see Eliat

Gasperini, M., \& Veneziano, G., Inflation, deflation, and frameindependence in string cosmology

Gerasimov, S.B., \& Khrykin, A.S., Can nucleon-nucleon bremsstrahlung shed more light on narrow di-nucleon structures?

Gerassimov, S.G., sec Adamovich

Ghosh, A., see Ghosh

Ghosh, D., Ghosh, A., Ghosh, P., Kundu, D. \& Roy, J., Intermittency in hadron-nucleus interactions at $200 \mathrm{GeV} / \mathrm{c}$ in terms of factorial correlators

Ghosh, G., see Dutta-Roy

Ghosh, P., see Ghosh

Ghoshal, D., \& Mahapatra, S., Three-point functions of nonunitary minimal matter coupled to gravity

Ghoshal, D., Lakdawala, P. \& Mukhi, S., Perturbation of the ground varicties of $c=1$ string theory

Gibilisco, M., see Alzetta

Giller, S., Gonera, C., Kosiński, P., Majewski, M., Maslanka, P. \& Kunz, J., On y-covariant wave functions

Gillman, T., see Elia

Gioumousis, A., ste' Elia

Gitman, D.M., \& Saa, A.V. Pseudoclassical model of spinning particle with anomalous magnetic momentum

Giulini, D., On the possibility of spinorial quantization in the Skyrme model

Gladding, G., see Elia

Goldman. T., sec Abluwalia

Goloskokov, S.V., Is the anomalous behavior of the pomeron spin-flip part possible in perturbative QCD'

Gomes, J.F., se' Aratyn

Gomes, M., sec Brunini

Gonera, C., sec Giller

González, P., \& Vento, V. Pionic effects in deep inelastic scattering off nuclei

González, $S$. seo Elia
A8 (1993) 787

A8 (1993) 21

A8 (1993) 21

A8 (1993) 2237

A8 (1993) 3701

A8 (1993) 2457

A8 (1993) 21

A8 (1.993) 3233

A8 (1993) 3233

A8 (1993) 1427

A8 (1993) 3233

A8 (1993) 197

A8 (1993) 3187

A8 (1993) 2335

A8 (1993) 3785

A8 (1993) 2237

A8 (1993) 2237

A8 (1993) 463

A8 (1993) 1917

A8 (1993) 2237

A8 (1993) 2623

A8 (1993) 3255

A8 (1993) 3079

A8 (1993) 1977

A8 (1993) 3785

A8 (1993) 1563

A8 (1993) 2237
González-Díaz, P.F., Quantum theory of wormholes

González-Díaz, P.F., Is there gauge quantum hair?

Gounaris, G., Renard, F.M. \& Schildknecht, D., Unitarity bounds on $W W$ scattering and electroweak tests at LEP

Gozzi, E., \& Reuter, M., Quantum-deformed geometry on phase-space

Grabowski, J., Marmo, G. \& Perelomov, A.M., Poisson structures: Towards a classification

Gracey, J.A., Gauged NambuJona-Lasinio model at $O(1 / N)$ with and without a ChernSimons term

Gràcia, X., \& Roca, J., Covariant and noncovariant gauge transformations for the conforma particle

Granovskii, Ya.I., \& Zhedanov,

A.S., Production of $Q$-boson by a classical current: an exactly solvable model

Greiner, W, see Dumitru

Greiner, W., see Spieles

Greiner, W., see Schönfeld

Grimus, W., \& Scharnagl, T., Neutrino propagation in matter and electromanetic fields

Grote, J., see Adamovich

Grundberg, J., Lindström, U. \&

Nordström, H., Discretization of the superparticle path integral

Guilarte, J.M., Fusion rules, topological quantum mechanics and three-manifolds

Gulamov, K.G., see Adamovich

Günaydin, M., Generalized conformal and superconformal group actions and Jordan algebras

Gupta, S.K., see Adamovich

Gupta, V.K., see Adamovich

Gurd, D.P., see Elia

Habib, S., see Fakir

Hadjiev, S.A., see Jatarov

Hagelin, J.S., Kelley, S. \& Tanaka, T., Exact supersymmetric amplitude for $K^{0}-K^{0}$ and $B^{0}-B^{0}$ mixing

Hale, D.L., see Elia

Haller, G.M., see Elia

Hallewell, G.D., sec' Elia

Halyo, E., Technidilaton or Higgs?

Hamilton, V., see Elia
A8 (1993) 1089

A8 (1993) 2539

A8 (1993) 647

A8 (1993) 1433

A8 (1993) 1719

A8 (1993) 2205

A8 (1993) 1747

A8 (1993) 1029

A8 (1993) 1291

A8 (1993) 2547

A8 (1993) 2631

A8 (1993) 1943

A8 (1993) 21

A8 (1993) 1323

A8 (1993) 3001

A8 (1993) 21

A8 (1993) 1407

A8 (1993) 21

A8 (1993) 21

A8 (1993) 2237

A8 (1993) 2827

A8 (1993) 237

A8 (1993) 2737

A8 (1993) 2237

A8 (1993) 2237

A8 (1993) 2237

A8 (1993) 275

A8 (1993) 2237 
Haney, M.J., see Elia Hansl-Kozanecka, T., see Elia Hansson, T.H., Karlhede, A. \& Westerberg, E., Anyons on higher genus surfaces - A constructive approach

Hargis, H., see Elia

Harris, M.G., \& Wheater, J.F., An improved metropolis algorithm for the simulation of random surfaces

Harrison, J., see Elia

Hart, E.L., see Elia

Hasegawa, K., see Elia

Hasegawa, Y., see Elia

Hayashi, M.J., see Fujishiro

Heckman, H.H., see Adamovich

Hedges, S., see Elia

Herscovitz, V.E., see Steffens

Hertzbach, S.S., see Elia

Hildreth, M.D., see Elia

Hiley, B., \& Monk, N., Quantum phase space and the discrete Weyl algebra

Hillion, P., The Bateman solutions of the spinor wave equation

Hilomen, R.C., see Elia

Himel, T.M., see Elia

Hiro-Oka, H., \& Minakata, H. Large- $N$ collective field theory applied to anyons in magnetic fields

Hiroshige, N., see Nagata

Hitlin, D.G., see Elia

Ho, C.-L., see Lee

Hodges, T.A., see Elia

Hodgson, J., see Elia

Hoeflich, J.J. see Elia

Hohler, E.G.B., \& Olaussen, K.. Conservation laws for the classical Toda field theories

Honecker, A., see Eholzer

Hong, W.-P., Cline, D.B. \& Jung

Y.-D., Detection of relic neutrinos from the decay of fourth generation neutrinos using the neutrino window on the earth

Honma, A., see Elia

Horelick, D., see Elia

Horiguchi, T., Third quantization of a solvable intlationary universe model with an exponential potential

Horvat, R, Electromagnetic forward neutrino-neutrino scattering in dense matter

Horváth, Z., see Duval

Horvathy, P.A., see Duval
A8 (1993) 2237 A8 (1993) 2237

A8 (1993) 3683 A8 (1993) 2237

A8 (1993) 1221

A8 (1993) 2237

A8 (1993) 2237

A8 (1993) 2237

A8 (1993) 2237

A8 (1993) 491

A8 (1993) 21

A8 (1993) 2237

A8 (1993) 3603

A8 (1993) 2237

A8 (1993) 2237

A8 (1993) 3625

A8 (1993) 2111

A8 (1993) 2237 A8 (1993) 2237

A8 (1993) 3547

A8 (1993) 1063

A8 (1993) 2237

A8 (1993) 1495

A8 (1993) 2237

A8 (1993) 2237

A8 (1993) 2237

A8 (1993) 3377 A8 (1993) 725

A8 (1993) 1461 A8 (1993) 2237 A8 (1993) 2237

A8 (1993) 777

A8 (1993) 3093 A8 (1993) 3749

A8 (1993) 3749
Hu, B.L., \& Zhang, Y.-H., Squeezed states and uncertainty relation at finite tem. perature

Hu, J.-G., \& Huang, C.-S., Conıments on $W^{ \pm}$and $Z^{0}$ decay to $\pi^{ \pm 0} \gamma$

Hu, S., see Huang

Huang, C.-S., see Hu

Huang, H., see Adamovich

Huang, Kai, \& Hu, S., $\theta$ statistic and topological properties in the $(2+1)$-dimensional CP model

Huang, Tau, sez Bian

Hübel, R., see Eholzer

Huber, J, see Elia

Huffer, M.E., see Elia

Hughes, E.W, see Elia

Hussein, M.S., see Frederico

Hussein, M.S., see Aleixo

Hwang, H., see Elia

Hyatt, E., see Eli

Igarashi, Y., see Fujiwara

Ignatiev, A.Yu.. see Dobroliubov Iida, K., Minakata, H. \& Yasuda, O., A test of the equivalence principle by long-baseline net rino-oscillation experiments

Ilinski, K.N., \& Uzdin, V.M.,

Quantum superspace, $q$ extended supersymmetry and parasupersymmetric quantum mechanics

Illuminati, F., Multi-anyon spectra and wave functions in the clustering approximation

Inagaki, I., Muta, T. \& Odintsov, S.D., Nambu-Jona-Lasinio model in curved space-time

lofa, M.Z., see Belokurov

Iohara, K., \& Malikov, F., Solutions to Knizhnik-Zamolodchikov equations with coefticients in non-bounded modules Irtan, M., see Alımad

Ishikawa, A., \& Isse, T., The stability of the minisuperspuce

Isse, T., Unification of gravity, gauge and Higgs fields by confined quantum tields - mathematical formulation

Isse, 'T., Unification of gravity, gauge and Higgs fields by confined quantum fields II - effective theory

Isse, T., see Ishikawa

Ito, M., Kisse, H. \& Moriti, K., Stochastic quantization of Nambu-Jona-Lasinio model
A8 (1993) 3575

A8 (1993) 459

A8 (1993) 3455

A8 (1993) 459

A8 (1993) 21

A8 (1993) 3455

A8 (1993) 635

A8 (1993) 725

A8 (1993) 2237

A8 (1993) 2237

A8 (1993) 2237

A8 (1993) 295

A8 (1993) 997

A8 (1993) 2237

A8 (1993) 2237

A8 (1993) 2147

A8 (1993) 917

A8 (1993) 1037

A8 (1993) 2657

A8 (1993) 513

A8 (1993) 2117

A8 (1993) 3345

A8 (1993) 3613 A8 (1993) 1103

A8 (1993) 3413

A8 (1993) 837

A8 (1993) 947 A8 (1993) 3413

A8 (1993) 903 
Itoh, Y., Higher order calculations in renormalization group approach to matrix models

Ivanov, A.N., see Faber

Ivanov, A.N., Troitskaya, N.I. Faber, M., Schaler, M. \& Nagy, M., On the hehavior of dynamical quark-antiquark pairs in external chromo-electric fields

Ivanov, A.N., Nagy, M. \& Troitskaya, N.I., The $K^{+} \rightarrow$ $\pi^{+} \pi^{\prime \prime} \gamma$ (DE) decay in chiral perturbation theory at the quark level

Iwasaki, Y., s'e $\epsilon^{\prime}$ Elia

Izen, J.M., see Elia

Jacques, P., see Elia

Jafarov, R.G., \& Hadjicv, S.A. Fermion-boson scattering in ladder approximation

Jagannathan, R., see Chakrabart Jagannathan, R., se' Chaturvedi

Jahns, A., se' Spieles

Jako, C.., se' Elia

Jakobsson, B., sec Adamovich

Jena, S.N., \& Panda, S., Quarkpion coupling constant in an independent-quark model with chiral symmetry

Jha, P.K., \& Tripathy, K.C. Symmetries of the renormalization group squations and the scaling law revisited

Jing, S., The Jordan-Schwinger realization of two-parametric quantum group $\mathrm{sI}_{\text {g }}(2)$

Jobe, R.K., se': Elia

Johnson. A.S., sec Elia

Johnson, J.R., s'e' Elia

Johnson, R.A., see Elia

Jones, S., s'c' Elia

Joo, K.G., ses Chung

Josti, K.G., s'e Dunbar

Judek, B., sc' Adumovich

Jue, C., ses Myung

Jung, Y.-D., se' Hong

Junk, ' $T$, se'e Elia

Junker, S., see Bancke

Just, L., see Adamovich

Kaclikachi, H., \& Kachkachi, $M$. Superconformal structures and holomorphic 1/2-superdifferentials on $N=1$ super Riemann surlaces

Kachkachi, M., see Kachkach

Kachroo, S., see Adamovich

Kaiser, S., see Elia

Kajikawa, R., sec Elia

Kalelkar, M., see Elia
A8 (1993) 3273

A8 (1993) 335

A8 (1993) 1021

A8 (1993) 1599 A8 (1993) 2237 A8 (1993) 2237

A8 (1993) 2237

A8 (1993) 237

A8 (1993) 2695

A8 (1993) 3727

A8 (1993) 2547

A8 (1993) 2237

A8 (1993) 21

A8 (1943) 607

A8 (1993) 3017

A8 (1993) 543

A8 (1993) 2237

A8 (1993) 2237

A8 (1993) 2237

A8 (1993) 2237

A8 (1993) 2237

A8 (1993) 2927

A8 (1993) 2803

A8 (1993) 21

A8 (1993) 341

A8 (1993) 1461

A8 (1993) 2237

A8 (1993) 2869

A8 (1993) 2

A8 (1993) 3643

A8 (1993) 3643

A8 (1993) 21

A8 (1993) 2237

A8 (1993) 2237

A8 (1903) 2237
Kaloper, N., A closed bianchi I universe in string theory

Kalyachkina, G.S., see Adamovich

Kang, H., see Elia

Kang, K., Knowles, I.G. \& White, A.R., The $\eta_{6}$ at LEP and TRISTAN

Kanygina, E.K., see Adamovich

Karabova, M., see Adamovich

Karlhede, A., see Hansson

Karliner, I., see Elia

Karlsen, R.E., Scadron, M.D. \& Bramon, A., SU(3) structure dependence in $\pi \rightarrow e v \gamma$ and $K \rightarrow e v \gamma$ decays

Kase, H., see Ito

Kashaev, R.M., \& Stroganov, Yu.G., Generalized Yang. Baxter equation

Kaul, G.L., see Adamovich

Kavalov, An.R., \& Mkrtchyan,

R.L., On a fermionic realizations of $W$-type symmetries

Kawahara, H., see Elia

Kawai, E.-I., A comment on the Hamilton formalism for nonlinear integrable models

Kawai, E.-I., A further comment on the Hamilton formalism for nonlinear integrable models

Keeler, R.K., see Elia

Kehagias, A.A., see Batakis

Kelley, S., Lopez, J.L. \& Nanopoulos, D.V., The scale where supersymmetry breaks: a new parameter in unified models

Kelley, S., see Hagelin

Kelsey, M.H., see Elia

Kendall, H.W., see Elia

Khadkikar, S.B., Parikh, J.C. \& Vinodkumar, P.C., Equation of state for quark gluon plasma in a relativistic harmonic confinement model

Kharakh, D., see Elia

Kharchev, S., Marshakov, A., Mironov, A. \& Morozov, A. Landau-Ginzburg topological theories in the framework of GKM and equivalent hierarchies

Khare, A., Mishra, A.K. \& Rajasekaran, G., Parasupersymmetric quantum mechanics and its infinite order limit

Khare, A., \& Kumar, C.N., Landau level spectrum for charged particle in a class of non-uniform magnetic fields
A8 (1993) 421

A8 (1993) 21

A8 (1993) 2237

A8 (1993) 1611

A8 (1993) 21

A8 (1993) 21

A8 (1993) 3683

A8 (1993) 2237

A8 (1993) 97

A8 (1993) 903

A8 (1993) 2299

A8 (1993) 21

A8 (1993) 3735

A8 (1993) 2237

A8 (1993) 1891

A8 (1993) 2919

A8 (1993) 2237

A8 (1993) 3057

A8 (1993) 177

A8 (1993) 2737

A8 (1993) 2237

A8 (1993) 2237

A8 (1993) 749

A8 (1993) 2237

A8 (1993) 1047

A8 (1993) 107

A8 (1993) 523 
Kharlamov, S.P., see Adamovich Khlebnikov, S.Yu., Disoriented chiral condensate in $(1+1)$ Lorentz-invariant geometry Khrykin, A.S., see Gerasimov Khudaverdian, O.M., \& Nersessian, A.P., On the geometry of the Batalin-Vilkovisky formalism

Kikukawa, Y., see Aoki

Kim, H.Y., see Elia

Kim J.K see Kim

Kim, K.Y., see Kim

Kim, K.Y., see Kim

Kim, P.C., see Elia

Kim, S.-K., see Kim

Kim, W.-T., see Kim

Kim, W.T., Shin, H. \& Kim J.K., Investigation of statisticschanging phase transition of Chern-Simons Higgs theory

Kim, W.-T., see Kim

Kim, Y., see Park

$\mathrm{Kim}, \mathrm{Y}$, see $\mathrm{Kin}$

Kim. Y., see Kim

Kim, Y.-W., Kim, S.-K., Kim, W.-T., Park, Y.-J., Kim, K.Y. \& Kim, Y., Lorentz invariant spectrum of minimal chiral Schwinger model

Kim, Y.-W., Park, Y.-J., Kim, K.Y., Kim, Y. \& Kim, W.-T., Realization of the Faddeevian regularization in an anomalous gauge theory

Kimura, K., \& Sugamoto, A., Renormalization group approach to the top condensation model and the $B^{0}-\bar{B}^{0}$ mixing

King, R., see Elia

Kirsten, K., see Bytsenko

Kiselev, V.V., Power correction to leading twist value of deep inelastic $R=\sigma_{l} / \sigma_{r}$

Kitakado, S., see Fujiwara

Kitazoe, T., see Anada

Kitroo, S., see Adamovich

Kizilersü, A., sece Akdeniz

Klauder, J.R., Coherent states without groups: Quantization on nonhomogeneous manifolds

Klein, M., see Elia

Krowles, I.G., see Kang

Kobayashi, T., Differential calculi on h-deformed bosonic and fermionic quantum planes

Kofler, R.R., see Elia

Kogan, I.I., se'e Dobroliubov
A8 (1993) 21

A8 (1993) 1901

A8 (1993) 2457

A8 (1993) 2377

A8 (1993) 3517

A8 (1993) 2237

A8 (1993) 3317

A8 (1993) 2155

A8 (1993) 3497

A8 (1993) 2237

A8 (1993) 2155

A8 (1993) 2155

A8 (1993) 3317

A8 (1993) 3497

A8 (1993) 417

A8 (1993) 2155

A8 (1993) 3497

A8 (1993) 2155

A8 (1993) 3497

A8 (1993) 1477

A8 (1993) 2237

A8 (1993) 2011

A8 (1993) 3799

A8 (1993) 1639

A8 (1993) 45,

$1065(\mathrm{E})$

A8 (1993) 21

A8 (1993) 2905

A8 (1993) 1735

A8 (1993) 2237

A8 (1993) 1611

A8 (1993) 3741

A8 (1993) 2237

A8 (1993) 2177
Koide, Y., Should the renewed tau mass value $1777 \mathrm{MeV}$ be taken seriously?

Kondo, K.-I., Tanabashi, M. \& Yamawaki, K., Renormalizing the gauged Nambu-JonaLasinio model

Kondo, K.-I., Gauge independent phase structure of gatuged Nambu-Jona-Lasinio and Yukawa models

Kosiński, P., see Giller

Kota, V.K.B., Eikonal scattering in the $s d g$ interacting boson model: analytical results in the $\mathrm{SU}_{\text {sg }}(3)$ limit and their generalizations

Kovner, A., Kurzepa, P. \& Rosenstein, B., A candidate for exact continuum dual theory for scalar $\mathrm{QED}_{3}$

A8 (1993) 2071

A8 (1993) 2859

A8 (1993) 3031 A8 (1993) 3785

A8 (1993) 987

A8 (1993) 1343 2527(E)

Kowitt, M., see Elia

A8 (1993) 2237

Krasnikov, N.V., The renormalization of the non-renormalizable four-dimensional four-fermion interaction

A8 (1993) 797

Krasnikov, N.V., On the calculation of the low energy soft supersymmetry breaking terms

Krasnikov, N.V., The influence of new physics on the value of strong coupling constant extracted from $Z$ total hadronic width

Krasnov, S.A., see Adamovich

Krasznovszky, S., Analysis of the KW distribution using a generalization of the Meijer's $G$-function

Krejcik, P., sec Elia

Krishna, N.M., see Elia

Kristjansen, C.F., see Ambjørn

Kroeger, R.S., see Elia

Kröger, H, Lantagne, S. \& Moriarty, K.J.M., Fraclal Wilson loop in U(1) lattice gauge theory

Kroha, H., Recent results from CLEO

Kubo, J., se' Fujiwara

Kudryavtsev, A.E., \& Tarasov V.E., Probabilities of the rare annihilation reactions $\bar{p} d \rightarrow$ $\pi^{-\prime} p, \rho^{-} p, \omega n, \eta n$ at rest

Kulikov, A.V., see Elia

Kulikova, S., see Adamovich

Kumar, A., sec Ali

Kumar, A., see Ali

Kumar, C.N., s'e Khare

\section{A8 (1993) 2579}

A8 (1993) 3483

A8 (1993) 21

A8 (1993) 483

A8 (1993) 2237

A8 (1993) 2875

A8 (1993) 2237

A8 (1993) 44.5

A8 (1993) 869

A8 (1993) 1005 A8 (1993) 22.37 A8 (1993) 21 A8 (1993) 1527 A8 (1993) 2045 A8 (1993) 523
A8 (1993) 2237 A8 (1993) 2147 
Kumar, V., see Adamovich

Kummer, W., \& Schwarz, D.J., Comment on "Canonical description of a two-dimensional gravity"

Kundu, A,, De, T. \& Dutta-Roy, B., Top quark mass in the condensate model and the possibility of colored bosons

Kundu, D., see Ghosh

Kuniba, A., Nakanishi, T. \& Suzuki, J., Characters in conformal field theories from thermodynamic bethe ansat

Kunz, J., se'e Giller

Kunz, P.F., see Elin

Kupershmidt, B.A., Sugawaratype constructions for the classical $W_{N}$ algebr

Kurzepa, P., see Kovner

Kwiatkowski, A., se'e Chetyrkin Kwiecinski, J, see Askew

Kwon, Y. see Elia

La, H.-S., Vortex solution in multi-Higgs system and its physical implication

Laartz, J., sece Forger

Labs, J.F., see Elia

Lahiri, A., The no-laair theoren for the Abelian Higgs model

Lahiri, A., Constrained dynamic: of the coupled Abelian twoform

Lakdawala, P., see Ghoshat

Lal, P., see Adamovich

Langstalf, R.R., sec Elid

Langston, M., sete Elia

Lantagne, S., sec Kröger

Larionova, V.G, see Adamovich

Larsen, R., see Elia

Lashkevich, M.Yu., Supercon formal 2D minimal models and an unusual coset construction

Lashkevich, M.Yu., Coset construction $\mathrm{SU}(2)_{k} \times \mathrm{SU}(2)$ $\mathrm{SU}(2)_{k+1}$ and minimal-like theories

Lath, A., sec Elia

Lauber, J.A., se' Elia

Lee, C.-C., \& Ho, C.-L., Symme

try breaking by Wilson lines and finite temperature and density effects

Lee, H.C., see Chiu

Leith, D.W.G., sec Elia

Lemke, E.H., A novel $\gamma W W$ vertex

Leonovich, A.A., \& Mladenov, D.M., Polynomialization of the Einstein-Hilbert action Leontaris, G.K., see Dreiner
A8 (1993) 21

A8 (1993) 2903

A8 (1993) 2465 A8 (1993) 3233

A8 (1993) 1649

A8 (1993) 3785

A8 (1993) 2237

A8 (1993) 1543 A8 (1993) 1343, $2527(\mathrm{E})$

A8 (1993) 2785

A8 (1993) 3813

A8 (1993) 2237

A8 (1993) 2649 A8 (1993) 803

A8 (1993) 2237

A8 (1993) 1549

A8 (1993) 2403

A8 (1993) 3187

A8 (1993) 21

A8 (1993) 2237

A8 (1993) 2237

A8 (1993) 445

A8 (1993) 21

A8 (1993) 2237

A8 (1993) 8.51

A8 (1993) 1243

A8 (1993) 2237

A8 (1993) 2237

A8 (1993) 1495

A8 (1993) 2911

A8 (1993) 2237

A8 (1993) 757

A8 (1993) 3251 A8 (1993) 2099
Lepetan, V.N., see Adamovich

Lew, H., see Fout

Lew, H., see Foot

Li, M., Some remarks on tachyon action in 2D string theory

Liberti, G., see Alzetta

Lima, J.A.S., \& Maia, J.M.F., Some cosmological consequences of a $\Lambda$-term varying as $\beta H^{2}+\alpha R^{-n}$

Limberg, T., see Elia

Lindström, U., see Grundherg

Lintern, L., see Elia

Liu, C, see Du

Liu, L.S., see Adamovich

Liu, X., see Elia

Lizzi, F., Marmo, G., Sparano, G. \& Vitale, P., Dynamical aspects of Lie-Poisson structures

Lokanathan, S., see Adamovich

Lopez, J.L., see Kelley

Lorant, S.St., see Elia

Lord, I., see Adamovich

Loreti, M., see Elia

Lowe, D.A., Conformal models of two-dimensional turbulence

Lozano, G., see Edelstein

Lu, A., see Elia

Lu, G.R., see Yue

Lukicheva, L.S., see Adamovich

Luo, S.B., see Adamovich

Luo, X.-Q., see Azcoiti

Lynch, H.L., sec Elia

Lyons, T., see Elia

Ma, J., see Elia

Ma, M. sce Awada

Ma, Z.-S., Adiabatic rotation for the skyrmions

Mackenzie, R., see Caenepeel

Maeda, K., see Fujiwara

Maekawa, N., see Bando

Maekawa, N., see Bando

Mahapatra, S., sez Ghoshal

Maharana, L., \& Nath, A.

Radiative transition $\Lambda_{c}^{+} \rightarrow \Sigma^{+} \gamma$

Maharana, L.., Nath, A. \& Panda, A.R., $B_{d}-\bar{B}_{d}^{0}$ mixing and top quark mass in a field theoretic quark model

Maharana, L., Nath, A. \& Panda, A.R., Weak leptonic decay of light and heavy pseudoscalar mesons in a field-theoretic quark model

Maia, J.M.F., see Lima

Maity, D.K., Banerjee, P.K., Das, B.B., Ravindran, D. \& Bhattacharjee, D.K., Intermittency patterns in proton-nucleus interactions at high energy
A8 (1993) 21

A8 (1993) 1859

A8 (1993) 3767

A8 (1993) 2481

A8 (1993) 2335

A8 (1993) 591

A8 (1993) 2237

A8 (1993) 1323

A8 (1993) 2237

A8 (1993) 2271

A8 (1993) 21

A8 (1993) 2237

A8 (1993) 2973

A8 (1993) 21

A8 (1993) 177

A8 (1993) 2237

A8 (1993) 21

A8 (1993) 2237

A8 (1993) 923

A8 (1993) 3665

A8 (1993) 2237

A8 (1993) 2843

A8 (1993) 21

A8 (1993) 21

A8 (1993) 3635

A8 (1993) 2237

A8 (1993) 2237

A8 (1993) 2237

A8 (1993) 2585

A8 (1993) 3569

A8 (1993) 1909

A8 (1993) 2147

A8 (1993) 2141

A8 (1993) 2729

A8 (1993) 197

A8 (1993) 399

A8 (1993) 1869

A8 (1993) 2775

A8 (1993) 591

A8(1993) 3853 
Majewski, M., see Giller

Majid, W,A., see Elia

Makeenko, Yu., Large- $N$ reduction, master field and loop equations in Kazakov-Migdal model

Makeenko, Yu., see Dobroliubov Maksimkina, T.N., see Adamovich

Malikov, F., see Iohara

Manashov, A.N., see Andrianov

Mancinelli, G., see Elia

Mancini, F., see Shanta

Mandal, G., see Dhar

Mandal, G., see Dhar

Mangano, G., \& Sannino, F., A mass spectrum for leptons and quarks

Mangazeev, V.V., \& Stroganov, Yu.G., Elliptic solution for modified tetrahedron equation

Mangotra, L.K., see Adamovich

Mani, H.S., see Bhawal

Manly, S., see Elia

Mansour, D., see Elia

Mansouri, F., \& Falbo-Kenkel

M.K., Reduction of the twobody problem with spin in $(2+1)$-dimensional gravity

Mantovani, G., see Elia

Maraner, P., \& Destri, C., Geometry-induced Yang-Mills fields in constrained quantum thechanics

Markiewicz, T.W., see Elia

Markin, O.Yu., Towards the gauged non-topological soliton

Marmo, G., see Grabowski

Marmo, G., see Lizzi

Marshakov, A., see Kharchev

Martellini, M., see Belgiorno

Martin, A.D., see Askew

Martina, L.. Pashaev, O.K. \&

Soliani, G., Self-dual Chern-

Simons solitons in nonlinear $\sigma$-models

Martínez-Morás, F., Mas, J. \& Ramos, E., Diffeomorphisms from higher-dimensional $W$ algebras

Marutyan, N.A., see Adamovich

Maruyama, T., see Elia

Mas, J., see Martínez-Morás

Maślanka, P., see Giller

Maslennikova, N.V., see Adamovich

Mason, G.R., see Elia

Masuda, H., see Elia

Mathur, V.S., see Das

Mathys, L., see Elia

Matsuda, M., see Nagata
A8 (1993) 3785 A8 (1993) 2237

A8 (1993) 209

A8 (1993) 2387

A8 (1993) 21

A8 (1993) 3613

A8 (1993) 2199

A8 (1993) 2237

A8 (1993) 1999

A8 (1993) 170

A8 (1993) 3557

A8 (1993) 1519

A8 (1993) 3475

A8 (1993) 21

A8 (1993) 1621

A8 (1993) 2237

A8 (1993) 2237

A8 (1993) 2503

A8 (1993) 2237

A8 (1993) 861

A8 (1993) 2237

A8 (1993) 2265

A8 (1993) 1719

A8 (1993) 2973

A8 (1993) 1047

A8 (1993) 2593

A8 (1993) 3813

A8 (1993) 3241

A8 (1993) 2189

A8 (1993) 21

A8 (1993) 2237

A8 (1993) 2189

A8 (1993) 3785

A8 (1993) 2

A8 (1993) 2237

A8 (1993) 2237

A8 (1993) 2079

A8 (1993) 2237

A8 (1993) 1063
Matsumura, Y., Sakai, N., Tanii, Y. \& Uchino, T., Correlation functions in two-dimensional dilaton gravity

Matsuo, Y., Some additional solutions of conformal turbulence

Matsuo, Y., Hopf term, loop algebras and three-dimensional Navier-Stokes equation

Mazaheri. G., see Elia

Mazzucato, A., see Elia

Mazzucato, E., see Elia

McArthur, I.N., \& Yung, C.M. Hirota bilinear form for the super-KdV hierarchy

McArthur, I.N., The partition function for the supersymmetric eigenvalue model

McCormick, D.J., see Elia

McDougall, N.A., see Dowrick

McGowan, J.F., see Elia

McHugh, S., see Elia

McKemey, A.K., see Elia

Meadows, B.T, see Elia

Medeiros, R.T., see Aratyn

Medvedev, P.B., see Aref'eva

Meenakumari, N., \& Rajasekaran, M., Non-topological soliton solutions for a dilaton type potential

Mellor, D.J., see Elia

Mendes, RS., see Brunelli

Merchant, A.C., see Rae

Messner, R., see Elia

Metsaev, R.R., Generating function for cubic interaction vertices of higher spin fields in any dimension

Migdal, A.A., 1/N expansion and particle spectrum in induced QCD

Migdal, A.A., Phase transitions in induced $O C D$

Migdal, A.A., Mixed model of induced $\mathrm{QCD}$

Migdal, A.A., Bose condensation and $Z_{N}$ symmetry breaking in the mixed model of induced QCD

Migdal, A.A., Exact solution of induced lattice gauge theory at large- $N$

Miller, H.G., see Yen

Minakata, H., see Iida

Minakata, H., see Hiro-Oka

Mincer, A.L., see Elia

Minic, D., see Chaudhuri

Minn, J., Scale independence of spinor anomaly

Minty, M., see Elia
A8 (1993) 1507

A8 (1993) 619

A8 (1993) 2677

A8 (1993) 2237

A8 (1993) 2237

A8 (1993) 2237

A8 (1993) 1739

A8 ( 1993$) 3355$

A8 (1993) 2237

A8 (1993) 503

A8 (1993) 2237

A8 (1993) 2237

A8 (1993) 2237

A8 ( 1993$) 2237$

A8 (1993) 3079

A8 (1993) 2167

A8 (1993) 667

A8 (1993) 2237

A8 (1993) 115

A8 (1993) 2435

A8 (1993) 2237

A8 (1993) 2413

A8 (1993) 139

A8 (1993) 153

A8 (1993) 245

A8 (1993) 259

A8 (1993) 359

A8 (1993) 1185

A8 (1993) 1037

A8 (1993) 3547

A8 (1993) 2237

A8 (1993) 276

A8 (1993) 121.5

A8 (1993) 2237 
Mironov, A., re Kharchev Mishra, A.K., see Khare Mittra, I.S., see Adamovich Mizumoto, Y., see Anada

Mkrtchyan, R.L., see Kavalov Mladenov, D.M., see Leonovich Mockett, P.M., see Elia

Moffeit, K.C., see Elia

Mohammedi, N., A large class of space-times from the $\operatorname{SL}(2, \mid$ F $) /$ U(1) coset model

Mohapatra, P.K., Differentiating various extra $Z$ 's at future collider:

Monk, N., sce Hiley

Montani, H., \& Wotzasek, C. Faddeev-Jackiw quantization of non-Abelian systems

Mookerjee, S., sec Adamovich

Moriarty, K.J.M., sec Kröger

Morita, K., see Ito

Morozov, A., se'c Kharchev

Morozov, A., \& Vinet, L., qhypergeometric functions in the formalism of free lields

Morrison, R.J., ser Elia

Mours, B., see Elia

Mueller, G., se' Eli

Mukli, S., see Choshal

Muller, D., see Elia

Mundy, G., se'e Elia

Muta, T., sce Enagaki

Myung, Y.S., Choi, J.M., I/n, M.J. \& Jue, C., Ground state perturbation of $N$-anyon in al magnetic field

Myung, Y.S., The chiral boson theory and edge states in the quantum Hall effect

Naculich, S.G.. Riggs, H.A. \& Schnitzer, H.J., Two-dimensional Yang-Mills theories are string theories

Natgamine, 'T., ste' Elia

Nagata, J., Yoshino, H. Malsuda, M., Hiroshige, N.\& Ueda. T., Two-pole analysis of narrow structures in KEK $m$ $A_{n}$, related with $p p-p p, \pi d-\pi i$ and $p p-\pi c d$ observables (Errata) Nagy, M., se' Faber

Nalgy, M., see Ivanov

Nagy, M, see Ivanov

Nahm, W., Recknagel, A. \& T'erhoeven, M., Dilogarithm identities in conformal field theory

Nakinishi, T., sec Kuniba

Nakano, H., se' Bandes

\begin{abstract}
A8 (1993) 1047
A8 (1993) 107

A8 (1993) 21

A8 (1993) 45 ,

$1065(\mathrm{E})$

Nakkagawa, H., Niégawa, A. \& Pire, B., Gauge independence of the fermion damping rate in a hot plasma - Landau gauge vs Coulomb gauge
\end{abstract}

A8 (1993) 3735

A8 (1993) 3251

A8 (1993) 2237

A8 (1993) 2237

A8 (1993) 1125

A8 (1993) 771

A8 (1993) 3625

A8 (1993) 3387

A8 (1993) 21

A 8 (1993) 445

As (1993) 903

A8 (1993) 1047

A8 (1993) 2891

A8 (1993) 2237

A8 (1993) 2237

A8 (1993) 2237

A8 (1993) 3187

A8 (1993) 2237

A8 (1943) 2237

A8 (1993) 2117

A8 (1993) 341

A8 (1993) 1297

A8 (1993) 2223

A8 (1993) 2237

A8 (1993) 1063

A8 (1993) 335

A8 (1993) 1021

A8 (1993) 1599

A.8 (1993) 1835

A8 (1993) 1649

A8 (1993) 2729
Nam, S., see Chung

Nanopoulos, D.V., see Kelley

Nasr, M.A., see Ahmad

Nasrulaeva, H., see Adamovich

Nasyrov, S.H., see Adamovich

Nath, A., see Maharan

Nath, A., see Maharana

Nath, A., see Maharana

Nauenberg, U., see Elia

Navotny, V.S., see Adamovich

Ndimubandi, J., A note on $q$ deformations and supersymmetric quantum mechanics

Neal, H., see Elia

Nelson, D., see Elia

Nersessian, A.P., see Khudaverdian

Nesterov, V., see Elia

$\mathrm{Ni}$, W.-T., see Wang

Niégawa, A., see Nakkagawa

Niemi, A.J., \& Palo, K., On quantum integrability and the Lefschetz number

Nikitin, A.G., see Beckers

Nojarov, R., \& Faessler, A., On the search for the scissors mode

Nojiri, S., \& Oda, I., Dilatonic supergravity in two dimensions and the disappearance of quantum black hole

Nordby, M., see Elia

Nordström, H., see Grundberg

Novikov, V.A., Okun, L.B. \& Vysotsky, M.I., Do present LEP data provide evidence for electroweak corrections'?

Nussbaum, M., see Elia

Nuttall, A., see Elia

Nystrand, $J$,, see Adamovich

O'Connor, D., Stephens, C.R. \& Freire, F. Dimensional reduc tion and the non-triviality of $\lambda \phi^{4}$ in four dimensions at high temperature

Oda, I., see Nojir

Odintsov, S., see Bytsenko

Odintsov, S.D., see Elizalde

Odintsov, S.D., see Antoniadis

Odintsov, S.D., see Bytsenko

Odintsov, S.D., see Inagaki

Odintsov, S.D., see Elizalde

Oehme, R., Singularities of hadronic amplitudes in quantum chromodynamics
A8 (1993) 739

A8 (1993) 2927

A8 (1993) 177

A8 (1993) 1103

A8 (1993) 21

A8 (1993) 21

A8 (1993) 399

A8 (1993) 1869

A8 (1993) 2775

A8 (1993) 2237

A8 (1993) 21

A8 (1993) 429

A8 (1993) 2237

A8 (1993) 2237

A8 (1993) 2377

A8 (1993) 2237

A8 (1993) 3715

A8 (1993) 739

A8 (1993) 2311

A8 (1993) 435

A8 (1993) 1171

A8 (1993) 53

A8 (1993) 2237

A8 (1993) 1323

A8 (1993) 2529 $3301(\mathrm{E})$

A8 (1993) 2237

A8 (1993) 2237

A8 (1993) 2

A8 (1993) 1779

A8 (1993) 53

A8 (1993) 2011

A8 (1993) 33

A8 (1993) 979

A8 (1993) 1131

A8 (1993) 2117

A8 (1993) 3325

A8 (1993) 1533 
Ogren, H., see Elia

Okun, L.B., see Novikov

Olaussen, K., see Hohler

Olsen, J., see Elia

Oram, C., see Elia

Orlova, G.I., see Adamovich

Osborne, L.S., see Elia

Oset, E., see Chiang

Osorio, M.A.R., \& Vázquez-

Mozo, M.A.. Variations on Kaluza-Klein cosmology

Osorio, M.A.R., \& Vázquez-

Mozo, M.A., String variations

on Kaluza-Klein cosmology

Ossa, R. see Elia

Otterlund, I., see Adamovich

Oxoby, G., see Elia

Pac, P.Y., \& Pahk, C.S., Strong $\mathrm{CP}$ problem and an axial $\mathrm{U}(1)$ gauge symmetry

Paccanoni, F., Stepanov, S.S. \& Tutik, R.S., Are the linear Regge trajectories really straight lines?

Paffrath, L., see Elia

Pahk, C.S., see Pac

Palo, K., see Niemi

Palounek, A., see Elia

Palsania, H.S., see Adamovich

Pan, S.-S., see Wang

Panda, A.R. see Maharana

Panda A.R see Maharana

Panda, A.R., \& Roy, K.C., Leptonic decay width of vector mesons in a field-theoretic quark model

Panda, S., see Jena

Panvini, R.S., see Elia

Panzeri. S., The $c=1$ matrix model formulation of two-dimensional Yang-Mills theories

Parashar, P., Bhasin, V.S. \& Soni, S.K. Covariant differential calculi on quantum symplectic and orthogonal planes

Parikh, J.C., see Khadkikar

Park, H., see Elia

Park, M.-I., Park, Y,-J. \& Kim, Y., Note on self-duality condition in the chiral boson theory

Park, Y.-J., see Park

Park, Y.-J., see Kim

Park, Y.-J., see Kin

Pashaev, O.K., se' $\epsilon^{\prime}$ Martina

Pashnev, A., see Chikalov

Patra, P.K. see Brahmachari

Pauluzzi, M., see Elia

Pavel, T J see Elia \begin{tabular}{l|c} 
A8 (1993) 2237 & Peleg, Y., Interacting third \\
A8 (1993) 2529, & quantized general relativity \\
$3301(E)$ & and change of topology \\
A8 (1993) 3377 & Peltoniemi, J.T., Singlet neutri- \\
A8 (1993) 2237 & nos as hot dark matter \\
A8 (1993) 2237 & Perelomov, A.M., see Grabowski \\
A8 (1993) 21 & Perelomov, A.M., \& Roan, S.-S., \\
A8 (1993) 2237 & A new example of $N=2$ \\
A8 (1993) 2259 & supersymmetric Landau-Ginz- \\
& burg theories: the two-ring
\end{tabular}

A8 (1993) 3111

A8 (1993) 3215

A8 (1993) 2237

A8 (1993) 21

A8 (1993) 2237

A8 (1993) 3049

A8 (1993) 549

A8 (1993) 2237

A8 (1993) 3049

A8 (1993) 2311

A8 (1993) 2237

A8 (1993) 21

A8 (1993) 3715

A8 (1993) 1869

A8 (1993) 2775

A8 (1993) 285

A8 (1993) 607

A8 (1993) 2237

A8 (1993) 320

A8 (1993) 389,

$2525(\mathrm{E})$

A8 (1993) 749

A8 (1993) 2237

A8 (1993) 417

A8 (1993) 417

A8 (1993) 2155

A8 (1993) 3497

A8 (1993) 3241

A.8 (1993) 285

A8 (1993) 1487

A.8 (1993) 2237

A8 (1993) 2237
A8 (1993) 1849

A8 (1993) 3593

A8 (1993) 1719

A8 (1993) 2989

A8 (1993) 21

A8 (1993) 3659

Peresadko, N.G., see Adamovic

Periwal, V., see Cohn

Perjés, Z., Parametric spinor approach to gravity

Perrier, F., see Elia

Peruzzi, I, see Elia

Pesando, I., see Cavicchi

Pesando, I., Vector induced lattice gauge theories

Pescara, L., see Elia

Peters, D., see Elia

Petersen, H., sec Elia

Petradza, M. s'e Elia

Petrov, N.V., see Adamovich

Phinney, N., see Elia

Piccolo, M., see Elia

Piemontese, L., see Elia

Pieroni, E., see Elia

Pire, B., see Nakkagawa

Pirogov, Yu.F., Vector boson dominance of electroweak interactions

Pitthun, R., see Elia

Pitts, K.T., see Elia

Plano, R.J., see Elia

Plyushchay, M.S., Pseudoclassical description of the massive spinning particle in $d$ dimensions

Plyushchev, V.A., see Adamovich

A8 (1993) 1969 A8 (1993) 2237

A8 (1993) 2237

A8 (1993) 2427. 2909(E)

A8 (1993) 2793

A8 (1993) 2237

A8 (1993) 2237

A8 (1993) 2237

A8 (1993) 2237

A8 (1993) 21

A8 (1993) 2237

A8 (1993) 2237

A8 (1993) 2237

A8 (1993) 2237

A8 (1993) 739

A8 (1993) 3129

A8 (1993) 2237

A8 (1993) 2237

A8 (1993) 2237

A8 (1993) 937

A8 (1993) 21

A8 (1993) 2237

Pollock, M.D., On the origin of density fluctuations in the universe

Popescu, S., see Aharonov

Porrati, M., see Ferrara

Prants, S.V., Lie-group treatment for two- and three-neutrino oscillations in matter with arbitrary density variations

Preparata, G., see Alzetta

Prepost, R., see Elia

Prescott, C.Y., see Elia

Pripstein, D., see Elia

Prodanov, E.M., see Arnaudov

Pulido, J.. Solar neutrinos with exotic scattering

Punithavelu, A.M., see Varma
A8 (1993) 1285

A8 (1993) 3691 A8 (1993) 2497

A8 (1993) 2671

A8 (1993) 2335

A8 (1993) 2237

A8 (1993) 2237

A8 (1993) 2237

A8 (1993) 1273

A8 (1993) 167
A8 (1993) 2943 
Punithavelu, A.M., see Varma

Punkar, G.D., see Elia

Putallaz, G., see Elia

Qarshiev, D.A., see Adamovich

Qian. W.Y., see Adamovich

Qin, Y.M., see Adamovich

Quano, Y.-H., \& Fujii, A., YangBaxter equation for broken $Z_{N}^{\otimes_{n}-1}$ models

Quano, Y.-H., Spontaneous polarization of the $\mathbb{Z}_{n}$-Baxter model

Quast, G., Recen results from LEP

Quesne, C., see Delbeed

Quesne, C., Realizations of a reflection quadratic algebra related to $\mathrm{SU}_{y}(N)$

Quick, R.M., see Yen

Rädel, G., \& Beyer, R., Neutrino electron scattering

Rae, W.D.M., \& Merchant, A.C., Shape eigenstates and other one- and two-dimensional at pha-cluster structures in light nuclei

Raimondi, P., see Elia

Rajasekaran, G., se'e Khare

Rajasekaran, M., sec Meenakumari

Rajeev, S.G., see Balaquic

Rajpoot, S., An SU(2) $\times U(1)_{\text {L }}$ $\mathrm{U}(1)_{\mathbb{R}}$ model with finite Dirac neutrino masses

Rajpoot, S., Massive Dirac neutrinos and SN I987A

Rama, S.K. \& Sen, S. Comments on Witten invariants of three-maanifokds for SU(2) and $Z_{m+1}$

Ramos, E., seer Martínez-Morás Raniwala, R, sec Adamovich

Raniwala, S., see Adamovich

Rankin, P., sece Elia

Rao, N.K., see Adiunovich

Rappoport, V.M., see Adamovich

Rashkov, R.Ch., see Arnaudov

Ratcliff, B.N., sce Elia

Ravindran, D., see Maity

Raychaudhuri, P., Time variation of solar neutrino flux

Recknagel, A., se' Nahm

Reeves, T.W. sec Elia

Rembieliński, J., wee Brzeziński

Rembieliński, J., \& Smoliński.

K.A., Non-conmutative quantum dynamics

Renard, F.M., sce' Gounaris

Rensing, P.E., se'e Elia

Reuter, M., sed Gorzi
A8 (1993) 3823

A8 (1993) 2237

A8 (1993) 2237

A8 (1993) 21

A8 (1993) 21

A8 (1993) 21

A8 (1993) 1585

A8 (1993) 3363

A8 (1993) 675

A8 (1993) 961

A8 (1993) 3467 A8 (1993) 1185

A8 (199.3) 1067

A8 (1993) 2435

A8 (1993) 2237

A8 (1993) 107

A8 (1993) 667

A8 (1993) 625

A8 (1993) 895

A8 (1993) 1179

A8 (1993) 2285

A8 (1993) 2189

A8 (1993) 21

A8 (1993) 21

A8 (1993) 2237

A8 (1993) 21

A8 (1993) 21

A8 (1993) 2943

A8 (1993) 2237

A8 (1993) 3853

A8 (1993) 1961

A8 (1993) 1835

A8 (1993) 2237

A8 (1993) 409

A8 (1993) 3335

A8 (1993) 647

A8 (1993) 2237

A8 (1943) 1433
Rhee, J.T., see Adamovich

Ricci, B., see Degl'Innocenti

Richman, J.D., see Elia

Riggs, H.A., see Naculich

Ring, P., see Gambhir

Rinta, R., see Elia

Rischke, D.H., see Dumitru

Roan, S.-S., see Perelomov

Robertson, L.P., see Elia

Roca, J., see Gràcia

Rochester, L.S., see Elia

Rohrlich, D., see Aharonov

Rosenson, L., see Elia

Rosenstein, B., see Kovner

Rosquist, K., \& Uggla, C., Visualizing minisuperspace dynamics

Ross, M.C., see Elia

Rosu, H., Black holes and radiometry

Rothberg, J.E., see Elia

Rothenberg, A., see Elia

Rowson, P.C., see Elia

Roy, J., see Ghosth

Roy, K.C., see Panda

Roy, P., see Barut

Rubakov, V.A., \& Semikoz,

D.V., Anomalous non-conservation of fermion quantum numbers in complex background gatuge fields: $(1+1)$-dimensional Abelian Higgs model

Rudaz, S., \& Srivastava, A.M. On the production of flux vortices and magnetic monopoles in phase transitions

Russell, J.J., see Elia

Rust, D., see Elia

Rutz, E., see Elia

Saa, A., On minimal coupling in Riemann-Cartan space-times

Saa, A.V., see Gitman

Saez., P., see Elia

Salu, P., Neutrino emissivity of degenerate diquark star matter

Sahu, P.K., Radial pulsation eigenfrequencies of diquark stals's

Saidkhanov, N., see Adamovich

Sakai, N., see Matsumura

Salnm, A., \& Sivaram, C., Strong gravity approach to QCD and confinement

Salcedo, L.L., see Arriola

Salmanova, N.A., see Adamovich

Samuel, M.A., The tau-lepton and tests of the standard model Sinnino, F., see Mangatno
A8 (1993) 21

A8 (1993) 471

A8 (1993) 2237

A8 (1993) 2223

A8 (1993) 787

A8 (1993) 2237

A8 (1993) 1291

A8 (1993) 2989

A8 (1993) 2237

A8 (1993) 1747

A8 (1993) 2237

A8 (1993) 3691

A8 (1993) 2237

A8 (1993) 1343

2527(E)

A8 (1993) 2815 A8 (1993) 2237

A8 (1993) 3429

A8 (1993) 2237

A8 (1993) 2237

A8 (1993) 2237

A8 (1993) 3233

A8 (1993) 2851

A8 (1993) 3507

A8 (1993) 1451

A8 (1993) 1443 A8 (1993) 2237 A8 (1993) 2237 A8 (1993) 22.37

A8 (1993) 2565

A8 (1993) 463

A8 (1993) 2237

A8 (1993) 3435

A8 (1993) 583

A8 (1993) 21

A8 (1993) 1507

A8 (1993) 32!

A8 (1993) 2061

A8 (1993) 21

A8 (1993) 2491

A8 (1993) 1519 
Santha, A.K., see Elia

Santocchia, A., see Elia

Sarkar, U., see Brahmachari

Sarkar, U., see Brahmachari

Sarkisova, L.G., see Adamovich

Sarkisyan, V.R., see Adamovich

Sato, J., see Bando

Savvidy, G.K., \& Savvidy, K.G., Gonihedric string and asymptotic freedom

Savvidy, K.G., see Savvidy

Saxton, O.H., see Elia

Sazdović, B., see Blagojević

Scadron, M.D., see Karlsen

Schaler, M., see Ivanov

Schalk, 'T., see Elin

Schaposnik, F.A., see Edelstein

Scharnagl, T., see Grimus

Schenk, P.R., see Elia

Schildknecht, D., see Gounaris

Schindler, R.H., see Elia

Schlumpf, F., The electric and magnetic form factors of the neutron

Schneekloth, U., see Elia

Schneider, M., see Elia

Schnitzer, H.J., see Naculich

Schönfeld, Th., Stöcker, H. Greiner, W. \& Sorge, H., Stopping power in nucleus-nucleus collisions at $\sqrt{s}=200 \mathrm{AGeV}$

Schröder, H., Recent results from ARGUS

Schuck, P., see Aouissat

Schultz, D., see Elia

Schultz, G.E., see Elia

Schumm, B.A., see Elia

Schwartz, A.J., Overview of rare and forbidden charm decays

Scliwarz, D.J., see Kummer

Seeman, J.T., see Elia

Seiden, A., see Elia

Selivanov, K.G., Geometry and physics on $w_{x_{s}}$ orbits

Semenoff, G.W., see Dobroliubov

Semenoff, G.W., see Dobroliubov

Semikoz, D.V., se' Rubakov

Sen, A., Magnetic monopoles, Bogomol'nyi bound and $\mathrm{SL}(2, \mathbb{Z})$ invariance in string theory

Sen, D., Semiclassical analysis of two- and three-spin antiferromagnets and anyons on a sphere

Sen, S., see Rama

Servoli, L., sec Elia
A8 (1993) 2237 A8 (1993) 2237 A8 (1993) 1487 A8 (1993) 3349

A8 (1993) 21

A8 (1993) 21

A8 (1993) 2729

A8 (1993) 2963

A8 (1993) 2963

A8 (1993) 2237

A8 (1993) 349

A8 (1993) 97

A8 (1993) 1021

A8 (1993) 2237

A8 (1993) 3665

A8 (1993) 1943

A8 (1993) 2237

A8 (1993) 647

A8 (1993) 2237

A8 (1993) 2135

A8 (1993) 2237

A8 (1993) 2237

A8 (1993) 2223

A8 (1993) 2631

A8 (1993) 573

A8 (1993) 1379

A8 (199.3) 2237

A8 (1993) 2237

A8 (1993) 2237

A8 (1993) 967

A8 (1993) 2903

A8 (1993) 2237

A8 (1993) 2237

A8 (1993) 1139

A8 (1993) 2177

A8 (1943) 2387

A8 (1993) 1451

A8 (1993) 2023

A8 (1993) 1805

A8 (1993) 2285

A 8 (109.3) 22.37
Settles, M., see Elia

Shabratova, G.S., see Adamovich

Shaevitz, M.H, see Elia

Shakhova, T.I., see Adamovich

Shank, J.T., see Elia

Shanta, P., Chaturvedi, S., Srinivasan, V. \& Mancini, F. Time-dependent Bogoliubov transformations and the damped harmonic oscillator

Shapiro, G., see Elia

Shapiro, S.L., see Elia

Shaw, H., s'e Elia

Shen, X., Quantum dilaton gravity in the light-cone gauge

Sheppard, J.C., see Elia

Sherden, D.J., see Elia

Shestakov, G.N., see Achasov

Shima, K., Spin-1/2 gauge field revisited

Shimomura, 'T., see Elia

Shin, H, see Kim

Shinke, H., \& Suzuki, H., Topological meaning of gauge anomaly and Gaussian factors

Shnir, Ya. M., se't Barut

Shore, G.M., \& Veneziano, G. The polarized photon structure function $g \gamma$ as a probe of chiral symmetry realizations

Shoup, A., see Elia

Shpilev, S.N., sec Adamovich

Shypit, R.L., see Elia

Siemann, R.H., s'c Elia

Simopoulos, C... see Elia

Sivaram, C. see Salam

Skarpaas, K., se' Elia

Skelding, D., ste Adumovich

Slaughter, M.D., A calculation of the magnetic moment of the $\Delta^{++*}$

Smith, S.R., see Elia

Smoliński, K.A., see Brzeziński

Smoliñski, K.A., se' Rentieliñski

Snyder, A., sece Elia

Snyder, J.A., se' Elial

Sobczyk, J., Current-current cor-

relation function on algebraic curves

Sobie, R., sce' Elia

Söderström, K., se" Adanovich

Soffer, J., sce Buccella

Sokolotif, M.D., s'e' Elia

Soliani, G., sec Martina

Solodukhin, S.N., Supersymmetric topological quantum field theories of differentia! forms I. Gauge p-forms
A8 (1993) 2237

A8 (1993) 21

A8 (1993) 2237

A8 (1993) 21

A8 (1993) 2237

A8 (1993) 1999

A8 (1993) 2237

A8 (1993) 2237

A8 (1993) 2237

A8 (1993) 697

A8 (1993) 2237

A8 (1993) 2237

A8 (1993) 2.343

A8 (1993) 2643

A8 (1993) 2237

A8 (1993) 3317

A8 (1993) 3835 A8 (1993) 3443

A8 (1993) 373

A8 (1993) 2237

A8 (1993) 21

A8 (1993) 2237

A8 (1993) 2237

A8 (1943) 2237

A8 (1993) 321

A8 (1993) 2237

A8 (1993) 21

A8 (1993) 3283 A 8 (199.3) 22.37 A8 (1993) $4(19)$

A8 (1943) 3335 A8 (1993) 2237 A8 (1993) 22,37

A8 (1993) 1153 A8 (1993) 22,37

A8 (1993) 21

A8 (1993) 225

A8 (1993) 2237

A8 (1993) 3241

A8 (1993) 2553 
Soloviev, O.A., Higgs conformal points from bosonized Thirring model

Soloviev, O.A., DashenFrishman conformal points for fermionic Thirring model from affine-Virasoro construction

Solovjeva, Z.I., see Adamovich

Sommerer, A.J., Spence, J.R. \& Vary, J.P., Generalized covariant two-body wave equation

Soni, S.K., see Parashar

Sorge, H., see Spieles

Surge, H., see Schönfeld

Sparino, G., sec Lizzi

Spence, J.R., see Sommerer

Spence, W.L., see Elia

Spencer, E.N., see Elia

Spieles, C., Jahns, A., Sorge, H.

Stöcker, H. \& Greiner, W.

Subthreshold antiproton pro-

duction in heavy-ion collisions

Sridhar, K., see Brahmachari

Sridhar, K., see' Brahmachari

Srinivasan, V., see Shanta

Srinivasan, V., sec Chaturved

Srivastava, A.M., see Rudaz.

Stamer, P., see Elia

Stanciu, S., ser FigueroaO'Farrill

Steffens, F.M. \& Herscovitz, V.E., Vibrational aspects of the SU(2) skyrmion

Steiner, H., sec Elia

Steiner, R., sec Elia

Steinhauser, M., scc Chetyrkin

Stenlund, E. see Adamovich

Stepanov, S.S., se'e Paccanoni

Stephens, C.R., se' O'Connor

Stephenson, R.J., see Elia

Stewart, G., see Elia

Stiles, P., see Elia

Stimptl-Abele, G., Kink finding in the aleph ТPC using neural networks

Stockdale, I.E., sece Elia

Stöcker, H., see Dumitru

Stiocker, H., sec' Spieles

Stöcker, H.. see Schönfeld!

Stoilov, M.N., \& Zaikov, R.P.

Quantum effects for generalized Chern-Simons type bosonic particle and string

Stoilov, M.N., \& Ziaikov, R.P. A U(1) Kac-Moody model coupled to 2D-pravity

Stratuss, M.G., se' Elia

Strugatnov, Yu.(i., sue Kashaev

Stroganov. Yu.di., se'e Mangateety
A8 (1993) 3071

A8 (1993) 21

A8 (1993) 3537

A8 (1993) 389 .

2525(E)

A8 (1993) 2547

A8 (1993) 2631

A8 (1993) 2973

A8 (1993) 3537

A8 (1993) 2237

A8 (1993) 2237

A8 (1993) 2547

A8 (1993) 1487

A8 (1993) 3349)

A8 (1993) 1999

A8 (1993) 3727

A8 (1993) 1443

A8 (1993) 2237

A8 (1993) 2125

A8 (1993) 3603

A8 (1993) 2237

A8 (1993) 2237

A8 (1993) 2785

A8 (1993) 21

A8 (1993) 549

A8 (1993) 1779

A8 (1993) 2237

A8 (1993) 2237

A8 (1993) 2237

A8 (1993) 2715

A8 (1993) 2237

A8 (1993) 1291

A8 (1993) 2547

A8 (1993) 26.31

A8 (1993) 313

A8 (1993) 2687

A8 (1993) 2237

A8 (1993) 2299

As $(1093,3475$
Su, D., see Elia

Suekane, F., see Elia

Sugamoto, A., see Kinura

Sugiyama, A., see Elia

Surin, E.L., see Adamovich

Sutton, P.J., see Askew

Suzuki, A.T., Evaluating one-

loop light-cone integrals by "covariantizing" the gauge-dependent pole

Suzuki, H., see Shinke

A8 (1993) 2237

A8 (1993) 2237

A8 (1993) 1477

A8 (1993) 2237

A8 (1993) 21

A8 (1993) 3813

A8 (1993) 2365

A8 (1993) 3835

A8 (1993) 1649

A8 (1993) 2237

Suzuki, S., see Elia

Svechnikova, L.N., see Adamovich

Swartz, M., see Elia

Szabo, R.J., see Dobroliubov

Szumilo, A., see Elia

Tabei, T., see Fujiwara

Tahar, M.Z. see Elia

Takahashi, T., see Elia

Takeshita, S., see Fujishiro

Takhtajan, L.A., Liouville theory: Quantum geometry of Riemann surfaces

Talebaoui, W., Theory of self-interacting scalar fields and gravitation

Tanabashi, M., see Kondo

Tanaka. T., see Hagelin

Tang, H., see Elia

Tanii, Y., see Matsumura

Tanii, Y.. \& Yamashita, M. Physical states in two-dimensional topological gauge theories

Tanimoto, M., Democralic quark mass matrix as an origin of ymmetric KM matrix

Tappern, G.J., sce Elia

Tarasov, V.E., see Kudryavtsev

Tarnopolsky, G., see Elia

Taylor, F.E., see Elia

Tecchio, M., see Elia

Teotonio-Sobrinho, P., se' Balachandran

Terao, H., \& Yamada, K. Equivalent models for gauged W7.W theory

Terhoeven, M., ste Nahm

Thaler. J.J., see Elia

Toevs, F., see Elia

Toge, N see Eli:

Tokarev, V.F., Does the strong CP-problem really exist?

Tolkachev, E.A., see Barut

Tolstov, K.D., see Adamovich

Torres, M., se'e Escalona

Tothovat, M., se' Adamovich

Tracas, N.D., se' Dreiner

Trelyalkovat, M.l., se'c Adamovich
A8 (1993) 21

A8 (1993) 2237

A8 (1993) 2177

A8 (1993) 2237

A8 (1993) 2147

A8 (1993) 2237

A8 (1993) 2237

A8 (1993) 491

A8 (1993) 3529

A8 (1993) 2053

A8 (1993) 2859

A8 (1993) 2737

A8 (1993) 2237

A8 (1993) 1507

A8 (1993) 1603

A8 (1993) 1387

A8 (1993) 2237

A8 (1993) 1005

A8 (1993) 2237

A8 (1993) 2237

A8 (1993) 2237

A8 (1993) 1305

A8 (1993) 2471

A8 (1993) 1835

A8 (1993) 2237

A8 (1993) 2237

A8 (1993) 2237

A8 (1993) 531

A8 (1993) 3443

A8 (1993) 21

A8 ( 1993$) 2955$

A8 (1993) 21

A8 (1993) 2094

A8 (1993) 21 
Triantaphyliou, G., QED radiative corrections to the decay $\pi^{0} \rightarrow e^{+} e^{-}$

Tripathy, K.C., see Jha

Trofimova, T.P., see Adamovich

Troitskaya, N.I., see Faber

Troitskaya, N.I. see Ivanov

Troitskaya, N.I., see Ivanov

Tsitsishvili, G., see Eliashvili

Tuleeva, U., see Adamovich

Turcotte, M., see' Elia

Turk, J.D., see Elia

Turner, J.L., sec Elia

Tutik, R.S., sce Paccanoni

Tyukhtyaev, Y.N., see Dvoeglazov

Tyutin, I.V., see Batalin

Tyutin, I.V., see Batalin

Ubriaco, M.R., Quantum deformations of quantum mechanics

Ubriaco, M.R., Quantum group Schrödinger field theory

Uchino. T. see Matsumura

Ueda, T., se'e Nagata

Uggla, C., see Rosquist

Um, M.J., see Myung

Usher, T., see Elia

Uzdin, V.M., ste llinski

Va'vra, J., see Elia

Valle Basagoiti, M.A., see Emparan

Vanderseypen, F.. Klein-GordonLangevin quantum geometry

Vandoren, S., se'e Aoyama

Vannini, C., see Elia

Varma, RK \& Plinithavelu, A.M., Behavior of charged particle motion along a magnetic field and a retarding potential-evidence for the existence of discrete energy states in classical mechanical domain

Varma, R.K.. \& Punithavelu. A.M., Behavior of charged particle motion in a magnetic field and a retarding potential II - observations of the existence of "forbidelen states" and Aharanov-Bohm analog in classical mechanical domain

Vary, J.P., see Sommerer

Vasiliev, M.A., se' Brink

Vasudevan, R, se' Chakrabarti

Vax, C., \& Witten, L., Reconstructing the metric of spacetime from Chern-Simons gravity with point sources (Erratum)

Vázquè-Mozo, M.A., se' Osorio

Vázquez-Mozo, M.A., see Osorio
A8 (1993) 1691

A8 (1993) 3017

A8 (1993) 21

A8 (1993) 335

A8 (1993) 1021

A8 (1993) 1599

A8 (1993) 3807

A8 (1993) 21

A8 (1993) 2237

A8 (1993) 2237

A8 (1993) 2237

A8 (1993) 549

A8 (1993) 3263 A8 (1993) 3673 A8 (1993) 3757

A8 (1993) 89

A8 (1993) 2213 A8 (1993) 1507 A8 (1993) 1063 A8 (1993) 2815 A8 (1993) 341 A8 (1993) 2237 A8 (1993) 2657 A8 (1993) 2237

A8 (1993) 3291

A8 (1993) 1671 A8 (1993) 3773 A8 (1993) 2237

A8 (1993) 167

A8 (1993) 3823 A8 (1993) 3537 A8 (1993) 358 A8 (1993) 2695

A8 (1993) 469)

A8 (1993) 3111

A8 (1993) 3215
Vella, E, see Elia

Veneziano, G., see Shore

Veneziano, G., see Gasperini

Vento, V., see González

Venuti, J.P., see Elia

Verdier, R., see Elia

Verdini, P.G., see Elia

Villalba, V.M., Dirac equation in

some homogeneous spacetimes, separation of variables and exact solutions

Villalba, V.M., Dirac spinor in a nonstationary Gödel-type cosmological universe

Vinet, L., see Mornzov

Vinodkumar, P.C., see Khadkikar

Visser, M., Hawking radiation: A particle physics perspective

Vitale, P., see Lizzi

Vladimirov, A.A., A method for obtaining quantum doubles from the Yang-Baxter $R$. matrices

Vladimirov, A.A., A closed ex pression for the universal $R$ matrix in a non-standard quantum double

Vokal, S., see Adamovich

Volkas, R.R., see Foot

Volovich, I.V., Affine strings

Vukašinac, T., see Blagojević

Vukašinac, T., see Antunović

Vysotsky. M.I., see Novikov

Wadia, S.R., see Dhar

Wadia, S.R., see Dhar

Wadsworth, B.F., see Elia

Waite, A.P., see Elia

Walker, N.J., se' Elia

Walz, D., see Elia

Wang, A. On the interaction of bubbles with gravitational and matter fields (Errata)

Wang, H.Q., sec Adamovich

Wang, S.-L., Ni, W.-T. \& Pan, S.-S., New experimental limi on the spatial anisotropy for polarized electrons

Warner, D., sec Elia

Watt, R., see Elia

Watts, S.J., sec' Elia

Weber, T., see Elia

Weidemann, A.W., sie Elia

Weigel, H., see Weiss

Weiss, C., Alkofer, R. \& Weigel, H., The Nambu-Jona-Lasinio soliton with generalized scalar interactions

Weng, Z.Q., see Adamovich

Westerberg, E., s'te Hansson
A8 (1993) 2237

A8 (1993) 373

A8 (1993) 3701

A8 (1993) 1563

A8 (1993) 2237

A8 (1993) 2237

A8 (1993) 2237

A8 (1993) 2351

A8 (1993) 3011

A8 (1993) 2891

A8 (1993) 749

A8 (1993) 1661 A8 (1993) 2973

A8 (1993) 1315

A8 (1993) 2573

A8 (1993) 21

A8 (1993) 1859

A8 (1993) 1827

A8 (1993) 349

A8 (1993) 1983

A8 (1993) 2529

$3301(\mathrm{E})$

A8 (1993) 1701

A8 (1993) 3557

A8 (1993) 2237

A8 (1993) 2237

A8 (1993) 2237

A8 (199.3) 2237

A8 (1993) 2907

A8 (1993) 21

A8 (1993) 3715

A8 (1993) 22.37

A8 (1993) 2237

A8 (1993) 2237

A8 (1993) 2237

A8 (1993) 2237

A8 (1943) 79

A8 (1993) 79

A8 (1993) 21

A8 (199.3) 3683 
Wexler, M., Critical behavior of target space mean field theory Wheater, J.F, see Harris Whitaker, J.S., see Elia

White, A.R., see Kang

White, S.L., see Elia

Wickens, F.J., see Elia

Wickert, S.A., see Elia

Wiese, K.J., Anomalous dimensions of soft operators in supersymmetric nonlinear sigma-models

Wilkes, R.J., see Adamovich

Williams, D.A., se' Elia

Williams, D.C., ses Elia

Williams, R.W., se'e Elia

Williams, S.H., see' Elia

Wilson, R.J., se' Elia

Wisniewski, W.J., se' Elia

Witherell, M.S., sec Elia

Witten, L., see Vaz.

Woodly, M.D., se' Elia

Woods, M., see Elia

Word, G.B., se' Eli:

Wotzasek, $C$., On the FaddeevJackiw quantization of the linear constraint chiral boson

Wotzasek, C., see Montani

Wu, Y.-L., A complete heavy quark effective Lagrangian from $Q C D$

Wyss, J., see Elias

Xia, Y.L., se' Adamovich

Xu, G.F., see Adamovich

Yamada, K., sec Tera

Yamada, Y., se' Eguchi

Yamaguchi, S.-1., Correlation functions of $(2 k-1,2) \mathrm{mini}$ mal matter coupled to $2 \mathrm{D}$ quantum gravity

Yamanoto, R.K. scee Elia

Yamartino, J.M., s'e Elia

Yamashita, M., se' Tanii

Yannawaki, K.. see Kondo

Yang, J.M., sece Yue

Yang, S.-K., sce Eguchi

Yang, Y.-P., \& Yu, Z.-R., qterwsor method of determining irreducible representation of quaritum algebra $\mathrm{SU}_{q}(3)$

Yac, Y.-P', we' Adel

Yasuda, (O., It'f' lida

Yee, C... se' Elia

Yellin, S.J., ste Lili
A8 (1993) 2703

A8 (1993) 122

A8 (1993) 2237

A8 (1993) 1611

A8 (1993) 2237

A8 (1993) 2237

A8 (1993) 2237

A8 (1993) 3845

A8 (1993) 21

A8 (1993) 2237

A8 (1993) 2237

A8 (1993) 2237

A8 (1993) 2237

A8 (1993) 2237

A8 (1993) 2237

A8 (1993) 2237

A8 (1993) 469

A8 (1993) 2237

A8 (1993) 2237

A8 (1993) 2237

A8 (1993) 2509 A8 (1993) 3387

A8 (1993) 819

A8 (1903) 2237

A8 (1993) 21

A8 (1993) 21

A8 (1993) 247 !

A8 (1993) 1627

A8 (1993) 327

A8 (1993) 22.37

A8 (1993) 2237

A8 (1993) 1603

A8 (1993) 2859

A8 (1993) 2843

A8 (1993) 1627

A8 (190)3 3025 A8 (1903) 1679) A8 (1993) 1037

A8 (1993, 2237

A8 (1993) 22.37
Yen, G.D., Miller, H.G. \& Quick, R.M., The origin of nuclear shape transitions Yeremian, A.D., see Elia

Yim, A., see Elia

Yoshino, H., see Nagata

Young, C.C., see Elia

Young, K.K. see Elia

Yu, Z.-R., sec Yang

Yue, C.X., Lu, G.R. \& Yang. J.M., Top-loop correction to neutral pseudo-Goldstone boson decays

Yung, C.M., The modified classical Yang-Buxter equation and supersymmetric Gel'fandDikii brackets

Yung, C.M., The $N=3$ supersymmetric KdV hierarchy

Yung, C.M., see McArthur

Yutit, H., see Elia

Zaikov. R.P.. sté Stoilov

Zaikuv, R.P., see Stoilov

Zapalac, G., see Elia

Zdarko, R.W. see Elia

Zeitlin, C., see Elia

Zeitlin. V.Y., Dense $(2+1)-\mathrm{di}$ mensional quantum electrodynamics in a uniform magnetic field

Zerbini, S., see Bytsenko

Zerbini, S., see Bytsenko

Zhang. D.H., se' Adamovich

Zhang, W.-M., \& Feng, D.H. Geometry in quantum non-integrability

Zhang, Y,-H., se'e Hu

Zhedanov. A.S. se' Granovskii

Zheng, P.Y., se' Adamovich

Zhokhova, S.I., sec Adamovich

Zhou, D.C. see Adamovich

Zhou, J., se'e' Elia

Ziemann, V., se'e Elia

Ziino, G., s'e' Barur

'Zimerman, A.H., se'e Aratyn

Zoller. D., see Awada

Zoller, V.R., Proton helicity flow to mesonic cloud and axial couplings of baryons

colotorev, M., 's'e' Elia

Tubarev, A.P., se Aref"eva

Zubarev, A.P., see Aref'eva

Zucchelli, P., see Elia
A8 (1993) 1185

A8 (1993) 2237

A8 (1993) 2237

A8 (1993) 1063

A8 (1993) 2237

A8 (1993) 2237

A8 (1993) 3025

A8 (1993) 2843

A8 (1993) 129

A8 (1993) 1161

A8 (1993) 1739

A8 (1993) 2237

A8 (1993) 313

A8 (1993) 2687

A8 (1993) 2237

A8 (1993) 2237

A8 (1993) 2237

A8 (1993) 1821

A8 (1993) 1131

A8 (1993) 1573

A8 (1993) 21

A8 (1993) 1417 A8 (1993) 3575

A8 (1993) 1029

A8 (1993) 21

A8 (1993) 21

A8 (1993) 21

A8 (1993) 2237

A8 (1993) 2237

A8 (1993) 1011

A8 (1993) 3079

A8 (1993) 2585

A8 (1993) 1113

A8 (1993) 2237

A8 (1993) 1469

A8 (1993) 2167

A8 (1993) 2237 